\title{
In vivo dissection of Rhoa function in vascular development using zebrafish
}

Laura M. Pillay ${ }^{1}$, Joseph J. Yano ${ }^{1,2}$, Andrew E. Davis ${ }^{1}$, Matthew G. Butler ${ }^{1}$, Keith A. Barnes ${ }^{1}$, Vanessa L. Reyes ${ }^{1}$, Daniel Castranova ${ }^{1}$, Aniket V. Gore ${ }^{1}$, Matthew R. Swift ${ }^{1}$, James R. Iben ${ }^{1}$, Amber N. Stratman ${ }^{1,3}$, and Brant M. Weinstein ${ }^{1^{*}}$

1. Division of Developmental Biology, National Institute of Child Health and Human Development, National Institutes of Health, Bethesda, MD, 20892

2. Present Address: Biology Department, University of Pennsylvania, 3400 Civic Center Boulevard, Philadelphia, PA 19104

3. Present Address: Department of Cell Biology and Physiology Washington University School of Medicine, St. Louis, MO 63110

${ }^{+}$Correspondence: Division of Developmental Biology, National Institute of Child Health and Human Development, National Institutes of Health, Bethesda, MD 20892

e-mail: flyingfish2@nih.gov

Short Title: Rhoa in regulating vascular development and integrity

Word Count: 10,992

Subject Codes: Developmental Biology, Vascular Biology, Angiogenesis, Genetically Altered and Transgenic Models, Intracranial Hemorrhage 


\section{ABSTRACT}

Rationale: The small monomeric GTPase RHOA acts as a master regulator of signal transduction cascades by activating effectors of cellular signaling, including the Rho-associated protein kinases ROCK1/2. Previous in vitro cell culture studies suggest that RHOA can regulate many critical aspects of vascular endothelial cell (EC) biology, including focal adhesion, stress fiber formation, and angiogenesis. However, the specific in vivo roles of RHOA during vascular development and homeostasis are still not well understood.

Objective: In this study we examine the in vivo functions of RHOA in regulating vascular development and integrity in zebrafish.

Methods and Results: We use zebrafish RHOA-ortholog (rhoaa) mutants, transgenic embryos expressing wild type, dominant-negative, or constitutively active forms of rhoaa in ECs, and a pharmacologic inhibitor of ROCK1/2 to study the in vivo consequences of RHOA gain- and lossof-function in the vascular endothelium. Our findings document roles for RHOA in vascular integrity, developmental angiogenesis, and vascular morphogenesis.

Conclusions: Our results indicate that either too much or too little RHOA activity leads to vascular dysfunction in vivo.

Key Words: Rhoa, endothelial cell, zebrafish, hemorrhage, angiogenesis 


\author{
Non-standard Abbreviations and Acronyms: \\ EC \\ endothelial cell \\ RHOA ras homolog gene family, member $\mathrm{A}$ \\ ROCK Rho-associated protein kinase \\ CCM cerebral cavernous malformation \\ hpf hours post fertilization \\ ENU N-ethyl-N-nitrosourea \\ SSLP Simple sequence length polymorphism \\ LDA lateral dorsal aortae \\ PHBC primordial hindbrain channel \\ CtA cranial central artery \\ DA dorsal aorta \\ CV cardinal vein \\ ISV intersegmental vessel \\ DN dominant negative \\ CA constitutively active \\ BBB blood brain barrier \\ HUVEC human umbilical vein endothelial cells \\ EGFP enhanced green fluorescent protein \\ UAS upstream activating sequence \\ 2A P2A viral cleavage peptide \\ Tg transgenic
}




\section{INTRODUCTION}

An endothelial cell (EC) monolayer forms the innermost layer of all blood vessels. This layer acts as a physical barrier to maintain blood vessel integrity and selective permeability, regulating the exchange of oxygen, solutes, nutrients, and immune cells between the blood and tissues. Under normal physiologic conditions, the EC barrier is maintained throughout the development of both embryonic and adult vascular networks, which form predominantly by angiogenesis - the "sprouting" of new vessels from pre-existing ones. Within the brain, disruption of the EC barrier leads to inflammation, edema, or hemorrhagic stroke, producing a spectrum of disease that includes neurologic dysfunction, disability, cognitive impairment, or death ${ }^{1}$. The formation, maintenance, and disassembly of EC barrier junctions are therefore highly regulated processes under strict spatiotemporal control ${ }^{2}$.

There are two principal types of barrier-forming EC junctions, tight junctions and adherens junctions 2, 3 . Tight junctions are continuous inter-EC junctions that limit paracellular permeability by controlling the exchange of cells, fluids, and solutes between the vessel lumen and surrounding tissues. Adherens junctions serve as anchors that maintain stable cellular associations under mechanical stress, or dynamic adhesive connections that facilitate cellular movement (e.g., during angiogenic blood vessel growth or remodeling). Both junction types are tethered to the actin cytoskeleton, and their assembly, maintenance and remodeling are modulated by actin-myosin contractile forces ${ }^{4}$. Although the intracellular signaling pathways that control EC cytoskeletal remodeling and junctional adhesion are still an active area of 
investigation, previous studies have implicated the Ras Homology (Rho)-family member RHOA in these processes (reviewed in ${ }^{2,4-7}$ ).

RHOA is a small, monomeric GTPase that transduces stimuli from hormones, growth factors, cytokines, and transmembrane proteins to downstream effectors of cellular signaling. RHOA acts as a molecular switch, cycling between a GDP-bound inactive state and a GTP-bound active state ${ }^{8,9}$. In response to upstream cellular signals, guanine nucleotide exchange factors (GEFs) switch RHOA to its active, GTP-bound state ${ }^{10}$. Active RHOA can then activate effectors including the Rho-associated protein kinases ROCK1 and ROCK2 ${ }^{11}$. GTPase-activating proteins (GAPs) inactivate RHOA by restoring it to its inactive, GDP-bound state ${ }^{10}$. Although previous studies suggest RHOA may regulate many critical aspects of vascular EC biology including cellular migration, proliferation, actin stress fiber formation, focal adhesion, and adherens junction dynamics 7, 12-16, the effects of RHOA on cytoskeletal dynamics and intercellular junction formation appear to be context-specific. Junction-destabilizing factors such as VEGF or thrombin 17-20, and junction-stabilizing factors such as Angiopoietin 1 or Sphingosine-1phosphate ${ }^{21-24}$ both appear to require RHOA and ROCK.

Due to the early embryonic lethality of Rhoa-knockout mice ${ }^{25}$ most of the functional characterization of RHOA in the vascular endothelium to date has been performed in cell culture, by overexpressing dominant negative or constitutively active forms of RHOA or by treating ECs in vitro with exogenous factors that modulate their growth and development ${ }^{4}$. ECs in culture are also typically grown under static growth conditions in the absence of other cell types. ECs in vivo are subject to continuous or pulsatile flow, producing laminar shear stress and cyclic stretch - forces that have been shown to differentially impact RHOA subcellular 
localization, signaling, and its effects on cytoskeletal remodeling ${ }^{26,27}$. ECs in vivo also interact with numerous other cell types that influence their behavior, including vascular smooth muscle cells, pericytes, macrophages, astrocytes, and neurons ${ }^{28}$. Consequently, although in vitro cell culture studies have been useful for identifying the cellular activities of RHOA in ECs, the in vivo biological significance of these studies remains unclear.

Studies of human patients and disease models with cerebral cavernous malformations (CCMs) have provided evidence that RHOA may play a role in regulating vascular integrity in vivo. CCMs are clusters of abnormally dilated blood vessels that exhibit vascular hyperpermeability and are extremely prone to rupture. Familial cases of CCM are associated with mutations in CCM complex genes (CCM1, 2, or 3$)^{29}$. The ECs of murine CCM models exhibit elevated RHOA and ROCK1/2 activity, along with a concomitant increase in actin stress fiber formation and reduced junctional integrity ${ }^{30-32}$. Treatment of murine CCM models with ROCK1/2 inhibitor decreases their hemorrhage and lesion burden ${ }^{31,33-35}$. CCM studies therefore suggest that RHOA signaling may negatively regulate vascular integrity in vivo. In support of this hypothesis, another study demonstrated that loss of EC RHOA in postnatal Cdh5-Cre ${ }^{\text {ERT2 }}$; Rhoof/f mice is sufficient to block vascular leakage following exogenous histamine but not VEGF-treatment ${ }^{36}$.

Other in vitro studies have also suggested roles for RHOA and ROCK1/2 in angiogenesis. RHOA and ROCK1/2 are required for VEGF-stimulated capillary formation from ECs in both in vitro cell culture and in mouse ex vivo retinal explants $15,37,38$. However, studies examining in vivo requirements for RHOA in developmental angiogenesis have produced conflicting reports. In one study, EC-specific deletion of Rhoa in Tie2-Cre; Rhoaf/f mice generated an embryonic lethal phenotype with vascular hypoplasia, yolk sac vascular remodeling defects, and dilated blood 
vessels, suggesting that EC-specific RHOA is required for proper vascular development and embryonic survival ${ }^{39}$. In contrast, a more recent study by a different group failed to observe any gross vascular abnormalities in their Tie2-Cre; Rhoaf/f EC-specific RHOA deficiency mouse model ${ }^{40}$. However, viable Tie2-Cre; Rhoa $^{f / f}$ pups were obtained at sub-Mendelian ratios in this study. It is therefore possible that EC RHOA deficiency produced an incompletely-penetrant, early embryonic-lethal phenotype in this model. Overall, existing studies of EC-specific RHOA function have provided limited, contradictory information on the requirement of EC RHOA in regulating embryonic vascular development in vivo.

Zebrafish provide a powerful model organism for studying embryonic vascular development and homeostasis. Forward and reverse genetic approaches in zebrafish have identified molecular pathways and genes that regulate vascular integrity and development and have clear orthologues in mammals ${ }^{41}$. Embryos develop externally, making them highly amenable to genetic manipulation. Optical transparency and transgenic strains permit visualization of hemorrhage and vascular development in vivo, in real-time through time-lapse microscopy ${ }^{42,43}$. The small size of zebrafish embryos allows them to survive and continue their development for many days in the absence of circulatory function, facilitating assessment of the specificity of vascular defects.

In this study, we use zebrafish as a model organism to assess the in vivo functions of Rhoa and its effectors Rock1/2 in regulating vascular development and integrity. We examine zebrafish with mutations in the zebrafish RHOA-ortholog rhoaa, transgenic animals with EC-specific expression of wild type or mutant forms of rhoaa, and animals treated with a pharmacological inhibitor of Rock1/2, using high-resolution optical imaging to study the consequences of Rhoa 
gain- and loss-of function in the vascular endothelium. Our results suggest that cranial vessel integrity in developing zebrafish is highly sensitive to either increased or decreased Rhoa gene dosage and activity, and that Rhoa and Rock1/2 are required for proper embryonic blood vessel growth and patterning in vivo. 


\section{METHODS}

\section{Data availability}

All data and materials reported in this manuscript are available from the Zebrafish International Resource Center (https://zebrafish.org) or upon request by contacting the corresponding author.

\section{Animal care and pharmacological treatments}

Fish were housed in a large zebrafish dedicated recirculating aquaculture facility (4 separate $22,000 \mathrm{~L}$ systems) in $6 \mathrm{~L}$ and $1.8 \mathrm{~L}$ tanks. Fry were fed rotifers and adults were fed Gemma Micro 300 (Skretting) once per day. Water quality parameters were routinely measured, and appropriate measures were taken to maintain water quality stability (water quality data available upon request). Zebrafish husbandry and research protocols were reviewed and approved by the NICHD Animal Care and Use Committee, in accordance with the Guide for the Care and Use of Laboratory Animals of the National Institutes of Health. The facility is accredited by the Association for Assessment and Accreditation of Laboratory Animal Care (AAALAC). EK strain zebrafish were used for all experiments unless otherwise noted. Embryos were grown at $28.5^{\circ} \mathrm{C}$ in embryo media (EM) or methylene blue-containing fish water ( $2 \mathrm{~mL}$ of $0.1 \%$ methylene blue per $1 \mathrm{~L}$ media) and staged according to standardized morphological criteria

44. EM was supplemented with $0.006 \%$ 1-phenyl 2-thiourea (PTU) (Sigma), to prevent pigment formation in post-24 hours post fertilization (hpf) embryos. Rockout (Rho Kinase Inhibitor III; Santa Cruz Biotechnology) was used to inhibit Rock1/2 activity ${ }^{45}$. Rockout was dissolved in Dimethyl sulfoxide (DMSO) to generate a stock concentration of $30 \mathrm{mM}$ and then diluted to a 
working concentration in EM + PTU. Embryos were treated from 24 hpf onward with $25 \mu \mathrm{M}$ or $50 \mu \mathrm{M}$ Rockout or equivalent dilutions of DMSO (solvent controls) in EM + PTU. All embryos were raised at $28.5^{\circ} \mathrm{C}$ and imaged or assessed for phenotypes at $52 \mathrm{hpf}$.

\section{Whole mount in situ hybridization}

Examination of gene expression by whole mount in situ hybridization was performed essentially as previously described 46,47 , with the following modifications: Riboprobes were designed to hybridize primarily to the $3^{\prime} U T R$ of each gene to minimize cross-reactivity between zebrafish orthologues with coding sequence similarities (see Table S1 for primer sequences used to amplify riboprobes). Prior to mRNA in situ hybridization analyses, embryos were fixed in $4 \%$ paraformaldehyde (PFA)/phosphate-buffered saline (PBS) overnight at $4^{\circ} \mathrm{C}$ or $4-5$ hours at room temperature (RT) with gentle agitation on a rotating platform. Embryos were permeabilized in $10 \mu \mathrm{g} / \mathrm{ml}$ proteinase $\mathrm{K}$ for 5 minutes (24-32 hpf embryos), or 30 minutes (48 $52 \mathrm{hpf}$ embryos) at RT.

\section{Transgenic zebrafish lines}

Published transgenic fish lines used in experiments include Tg(gata1:DsRed $)^{\text {sd2Tg }}{ }^{48}$, $T g(k d r l: E G F P)^{s 84349}, T g(f l i: E G F P)^{y 150}$, and $T g(e g f l 7: G A L 4 F F)^{g S A l z G F F D 478 A 51,52 .}$

All cloning was performed using SLiCE cloning techniques unless otherwise noted ${ }^{53}$. See Table S1 for primer sequences used for SLiCE cloning. The p14xUAS-Tom-2A plasmid was made by cloning the Tomato fluorescent protein (Tom) and P2A viral cleavage peptide (2A) 54,55 sequences into the multiple cloning site (MCS) of pT1ump-14xUAS-MCS-POUT (pT1UMP), which includes an E1b basal promoter, the Ocean pout antifreeze protein polyA, and is flanked by Tol1 
arms ${ }^{56}$. Select rhoaa coding sequences were PCR-amplified from wild type or rhoaa-mutant $(\mathrm{R} 17, \Delta 3)$ zebrafish cDNAs and cloned into pCRII-TOPO (Invitrogen), according to manufacturer's specifications. To make p14XUAS-Tom-2A-rhoaa, p14XUAS-Tom-2A-rhoaaR17, and p14XUASTom-2A-rhoaa $\Delta 3$, rhoaa coding sequences were PCR-amplified from wild type or rhoaa-mutant / pCRII-TOPO template and cloned into p14xUAS-Tom-2A. p14XUAS-Tom-2A-rhoaaN19 and p14XUAS-Tom-2A-rhoaaV14 were made by site-directed mutagenesis of p14XUAS-Tom-2Arhoaa. G0 Tg(UAS-Tom-2A), Tg(UAS-Tom-2A-rhoaa), Tg(UAS-Tom-2A-rhoaaR17), Tg(UAS-Tom2A-rhoaaA3), Tg(UAS-Tom-2A-rhoaaN19), and Tg(UAS-Tom-2A-rhoaaV14) transgenic zebrafish embryos were each generated by injecting individual p14xUAS-Tom-2A or p14xUAS-Tom-2Arhoaa isoforms into zebrafish embryos along with zebrafish-optimized Tol1 transposase RNA ${ }^{57}$.

\section{Mutant zebrafish lines and genotyping analyses}

The $y 172$ (aka R17, rhoaa ${ }^{R 17}$, or rhoaa ${ }^{y 172}$ ) mutant was identified in an F2 ENU mutagenesis screen for dominant mutants displaying a hemorrhage phenotype at 2-4 dpf in the $T g(f l i: E G F P)^{y 1}$ background (Figure S1). Bulked segregant analysis and fine genetic mapping of y172 were carried out as described previously ${ }^{58}$ and linked y172 to a $3.148 \mathrm{MB}$ region on Chromosome 8, in between determined SSLP markers ZC221E5-6 and ZK83F4-7 (Figure S1). Whole genome sequencing (Illumina HiSeq)-based identification of deleterious mutations within the critical mapping region revealed a p.G17R mutation in rhoaa. The rhoaa ${ }^{y 172}$ or rhoaa $^{R 17}$ mutants were genotyped using derived cleaved amplified polymorphic sequence (dCAPS) analysis ${ }^{59-61}$. Briefly, genomic DNA was isolated from adults or embryos as previously described ${ }^{62}$. PCR was used to introduce an Xbal restriction enzyme site in R17-mutant, but not WT rhoaa PCR product, using rhoaaY172Geno-F and rhoaaY172Geno-R primers (Table S1). PCR 
products were cleaved with Xbal, generating 194 bp WT and/or 169 bp R17-mutant products, analyzed by gel electrophoresis.

rhoad $^{\Delta 3}$ mutants were generated using CRISPR/Cas9 technology as previously described ${ }^{63}$, using the single-guide RNA sequence ATCGTTGGAGATGGAGCCTGTGG, which was selected using CHOPCHOP (https://chopchop.cbu.uib.no) ${ }^{64}$ to target the Rhoaa GTP/GDP binding domain. rhoaa ${ }^{\Delta 3}$ mutant embryos and adults were genotyped as previously described ${ }^{65}$. Genomic DNA was extracted using the REDExtract-N-Amp Tissue PCR Kit (Sigma): $50 \mu l$ of extraction solution and $12.5 \mu \mathrm{l}$ of tissue preparation solution was added to each embryo. Samples were mixed by vortex, incubated $10 \mathrm{~min}$ at RT, vortexed, and heated to $95^{\circ} \mathrm{C}$ for $4 \mathrm{~min}$. $50 \mu \mathrm{l}$ of neutralization solution B was added, samples were vortexed, and stored at $-80^{\circ} \mathrm{C}$ or used immediately. Solution volumes were doubled for DNA extraction from adult tail clips. Gene-specific, tailed genotyping primers (rhoaaM13Ftail-F and rhoaaPigtail-R, Table S1) were designed to amplify a $184 \mathrm{bp}$ fragment spanning the CRISPR binding site. A $10 \mu$ l total volume AmpliTaq-Gold (Life Technologies) PCR reaction was set up for each sample, containing $1 \mu$ l0X PCR Gold Buffer, $0.5 \mu \mathrm{l} 25 \mathrm{mM} \mathrm{MgCl}$, $1 \mu \mathrm{l} 0.5 \mathrm{mM}$ rhoaaM13Ftail-F primer, $1 \mu \mathrm{l} 1 \mathrm{mM}$ rhoaaPigtail-R primer, $0.2 \mu \mathrm{l} 10$ mM FAM-M13 primer, $0.1 \mu \mathrm{l} 10$ mM each dNTP Master Mix, 0.1 $\mu \mathrm{l}$ TaqGold polymerase, $1 \mu \mathrm{l}$ of a 1:10 dilution of genomic DNA in water, and $5.1 \mu$ water. PCR conditions were as follows: 10 min denaturation at $95^{\circ} \mathrm{C}$; 35 cycles of $95^{\circ} \mathrm{C}$ for $30 \mathrm{sec}, 58^{\circ} \mathrm{C}$ for $30 \mathrm{sec}$, and $72^{\circ} \mathrm{C}$ for $30 \mathrm{sec}$; and $10 \mathrm{~min}$ final extension at $72^{\circ} \mathrm{C} .2 \mu \mathrm{l}$ of PCR product was combined with $0.2 \mu \mathrm{l}$ ROX400HD (Life Technologies) and $9.8 \mu \mathrm{l} \mathrm{HiDi} \mathrm{Formamide,} \mathrm{and} \mathrm{the}$ solution was denatured at $95^{\circ} \mathrm{C}$ for $5 \mathrm{~min}$ and run on a ABI3130XL Genetic Analyzer (Applied Biosystems), using GeneMapper software (Life Technologies). 


\section{Endothelial cell culture}

Passage 1-6 human umbilical vein endothelial cells (HUVEC, Lonza) were cultured in 0.003\% Endothelial cell growth supplement (Millipore), 0.01\% Heparin, 20\% FBS, $2.5 \mathrm{ug} / \mathrm{mL}$ Amphotericin B and 0.1X penicillin/streptomycin in M199 media (Gibco) on $1 \mathrm{mg} / \mathrm{ml}$ gelatin coated tissue culture flasks at $37{ }^{\circ} \mathrm{C}$ in a humidified $5 \% \mathrm{CO} 2$ incubator, as previously described 66.

Lentiviral constructs for use in HUVEC in vitro were generated using SLiCE cloning techniques unless otherwise noted ${ }^{53}$. See Table S1 for primer sequences. Constructs with the CMV promoter were used because the PGK promoter has previously been shown to exhibit reduced or absent activity in endothelial cells, including HUVEC ${ }^{67,68}$. The sCMV promoter was amplified from pCS2+ ${ }^{69}$ by PCR and cloned into the pCDH-EF1 $\alpha$-MCS-(PGK-GFP-T2A-PURO) bidirectional promoter lentiviral vector (System Biosciences), replacing the PGK promoter, to generate pCDH-EF1 $\alpha$-MCS-(sCMV-GFP-T2A-PURO). Wild type or mutant $(R 17, N 19, V 14, \Delta 3)$ rhoaa coding sequences were PCR-amplified from p14XUAS-Tom-rhoaa constructs and cloned into the MCS of pCDH-EF1 $\alpha$-MCS-(sCMV-GFP-T2A-PURO) to generate pCDH-rhoaa (WT, R17, N19, V14, $\Delta 3$ ). Tomato (Tom) fluorescent protein sequences were then PCR-amplified and cloned into the MCS of pCDH-EF1 $\alpha$-MCS-(sCMV-GFP-T2A-PURO) or into pCDH-rhoaa to generate $\mathrm{pCDH}$-Tom control or pCDH-Tom-rhoaa constructs. Notably, previous studies indicate that $\mathrm{N}$-terminal epitope or fluorophore-tagging does not significantly impact RHOA subcellular localization or activity in endothelial cells ${ }^{70,71}$. 
Lentivirus was made from pCDH-Tom and pCDH-Tom-rhoaa (WT, R17, $\Delta 3$, N19, or V14) using the ViraPower Lentiviral Expression System (Invitrogen): $9 \mu \mathrm{g}$ ViraPower Packaging Mix and $3 \mu \mathrm{g}$ plasmid were added to $1.5 \mathrm{~mL}$ Opti-MEM. $36 \mu \mathrm{l}$ Lipofectamine 2000 was added to $1.5 \mathrm{~mL}$ OptiMEM. Solutions were gently mixed, incubated 5 minutes ( $\mathrm{min})$ at room temperature (RT), then combined, gently mixed, and incubated $20 \mathrm{~min}$ at RT. The resulting solution was added dropwise to a T75 flask of $90 \%$ confluent HEK293T cells grown in $10 \%$ FBS in DMEM media (Gibco) and gently mixed by tilting. Cells were incubated at $37{ }^{\circ} \mathrm{C}$ in a humidified $5 \% \mathrm{CO} 2$ incubator, and inspected for syncytia formation and Tomato/GFP-fluorescence. Cell media was replaced daily. Lentivirus-containing media was collected at both 2 and 3 days posttransfection: Cell debris was pelleted by centrifugation $15 \mathrm{~min}$ at 3,000 rpm and discarded. Virus-containing media was filtered through a Millex-HA $0.45 \mu \mathrm{m}$ filter and stored at $-80{ }^{\circ} \mathrm{C}$ until use.

Stable HUVEC lines expressing Tomato, Tomato-rhoaa, Tomato-rhoaaR17, Tomato-rhoaa ${ }^{\Delta 3}$, or Tomato-rhoaa ${ }^{\mathrm{N} 19}$ from integrated lentiviral vectors were generated by infecting HUVEC with the appropriate Lentivirus: $2.5 \mu \mathrm{l} 6 \mathrm{mg} / \mathrm{mL}$ Polybrene was mixed with $500 \mu \mathrm{l}$ of HUVEC growth media. Media was removed from 90\% confluent HUVEC grown in 6-well tissue culture plates, and Polybrene mixture was added dropwise to cells. $1 \mathrm{~mL}$ of Lentivirus-containing media was added to culture, and HUVEC were grown at $37{ }^{\circ} \mathrm{C}$ until greater than $30 \%$ expressed GFP. Transgene-expressing HUVEC were selected by Puromycin treatment. We were unable to recover stable lines of HUVEC expressing Tomato-rhoaa ${ }^{\mathrm{V} 14}$ due to non-viability of cells. 


\section{Immunohistochemistry}

HUVEC were transferred to $1.33 \%$ collagen / phosphate-buffered saline (PBS)-coated Lab-Tek chamber slides (ThermoFisher) at $90 \%$ confluency and allowed to attach overnight at $37^{\circ} \mathrm{C}$. Cells were fixed for 5 min in $100 \%$ methanol at RT. All washes and incubations were performed with gentle agitation on a rotating platform at RT, unless otherwise indicated. Cells were washed 4 x 5 min in tris-buffered saline (TBS; $136 \mathrm{mM} \mathrm{NaCl}, 25 \mathrm{mM}$ Tris, pH 7.4), then $15 \mathrm{~min}$ in tris-glycine buffer (0.025M Tris, $0.192 \mathrm{M}$ glycine, $\mathrm{pH} 8.3)$. Cells were permeabilized $30 \mathrm{~min}$ in 0.1\% Saponin/TBS, washed $4 \times 5 \mathrm{~min}$ in TBS, and blocked 1 hour in TBS $+0.1 \%$ Tween-20 (TBST) $+2.5 \%$ bovine serum albumin (BSA) $+5 \%$ sheep serum, then incubated in at 1:500 dilution of anti-VE Cadherin antibody (abcam, ab33168) in blocking solution, overnight at $4{ }^{\circ} \mathrm{C}$. Cells were washed $4 \times 5$ min in TBST, incubated 1 hour in a 1:1000 dilution of Alexa Fluor 488-conjugated secondary antibody (ThermoFisher) in blocking solution, then incubated in a 1:2000 dilution of Hoechst 33342/TBS solution (Invitrogen) for $20 \mathrm{~min}$. Cells were washed 4 × 5 min in TBST, mounted in Fluorescent Mounting Medium (Dako), and stored at $4^{\circ} \mathrm{C}$ in the dark.

\section{Western analysis}

Zebrafish crude protein lysates were prepared by adding $1 \mathrm{~mL}$ cold deyolking buffer (110 mM $\mathrm{NaCL}, 5.5 \mathrm{mM} \mathrm{KCl}, 10 \mathrm{mM}$ Tris $\mathrm{pH} 8.5,2.7 \mathrm{mM} \mathrm{CaCl} 2)$ containing cOmplete Mini Protease Inhibitor Cocktail (Roche) to 20 - 30 embryos. Yolk was disrupted by gentle pipetting followed by $30 \mathrm{sec}$ of $1100 \mathrm{rpm}$ vortex. Tissue was pelleted by centrifuging $1 \mathrm{~min}$ at 3,000 rpm and supernatant was discarded. $0.7 \mathrm{~mL}$ of cold wash buffer $(110 \mathrm{mM} \mathrm{NaCl}, 5.5 \mathrm{mM} \mathrm{KCl}, 10 \mathrm{mM}$ Tris $\mathrm{pH}$ 8.5, $2.7 \mathrm{mM} \mathrm{CaCl}$ ) containing protease inhibitor was added. Tissues were subjected to 
Dounce homogenization, centrifuged $5 \mathrm{~min}$ at 5,000 rpm and the supernatant discarded. $5 \mu \mathrm{l}$ of 2X LDS Sample Buffer containing $5 \%$ 2-Mercaptoethanol per embryo was added to each sample. Lysates were vortexed, incubated $10 \mathrm{~min}$ at $85^{\circ} \mathrm{C}$, then stored at $-20^{\circ} \mathrm{C}$.

Immediately prior to analysis, a 1:2 dilution of lysate to 1X LDS Sample Buffer was prepared and heated $10 \mathrm{~min}$ at $85^{\circ} \mathrm{C} .15 \mu \mathrm{l}$ of diluted lysate was run out on NuPAGE Novex Bis-Tris gels using the XCell Sure Lock Mini-Cell system (Invitrogen) at $140 \mathrm{~V}$, according to manufacturer's specifications. Proteins were transferred to methanol-treated PVDF membranes using a Mini Trans-Blot Cell wet-transfer apparatus (BIO-RAD), according to manufacturer's specifications at $400 \mathrm{~mA}$ for 1.5 hours. All membrane washes and incubations were performed with gentle rocking. Following transfer, membranes were washed 5 min in Tris-buffered saline with $0.1 \%$ Tween-20 (TBST), then allowed dry completely. Membranes were rehydrated in methanol, rewashed with TBST, and blocked at least $1 \mathrm{hr}$ in 5\% skim milk in TBST. Membranes were incubated in primary antibody diluted in TBST (1:500 anti-RHOA antibody, Cytoskeleton ARH04) or diluted in block (1:30,000 anti-a-alpha tubulin antibody, Sigma T6199) overnight at $4{ }^{\circ} \mathrm{C}$. After 5 × 5 min RT TBST washes, membranes were incubated in 1:2000 digital HRP-conjugated secondary antibody (Kindle Bioscience) in TBST. After TBST washes, a 1-Shot Digital ECL reaction (Kindle Bioscience) was performed, and images were acquired using a KwikQuant Imager (Kindle Bioscience). Quantification of relative band density was performed using ImageJ software ${ }^{72}$. Data is reported as the percent average density from a minimum of 3 blots from independent experiments \pm standard deviation. 


\section{Microscopy and imaging}

Fluorescent images of HUVEC were acquired using a Zeiss LSM 880 confocal microscope with Fast Airyscan, using a $63 \mathrm{X}$ objective with optical zoom. All images were acquired using the same settings.

Whole-mount in situ hybridization images and transmitted-light images of live zebrafish embryos were collected using a Leica M205 stereo microscope and LAS V4.7 software, with extended depth of focus z-stack image processing. Fluorescent images of zebrafish embryos were collected using a Nikon Yokogawa CSU-W1 spinning disk confocal microscope. Transmitted light movies of live zebrafish embryos were collected using a Leica M205 stereo microscope and LAS V4.7 software, or a Nikon Yokogawa CSU-W1 spinning disk confocal microscope. Prior to imaging, live embryos were immobilized in EM containing buffered MS222 and embedded in $0.8 \%$ low melting point agarose in EM. Zebrafish embryos were genotyped after imaging and data analysis.

All fluorescent images were processed using ImageJ ${ }^{72}$ or Imaris (Oxford Instruments) and all figures were assembled using Powerpoint (Microsoft) and/or Photoshop (Adobe). Copies of original transmitted light images of live zebrafish embryos were globally adjusted for red color saturation to enhance red blood cell visibility equally across matched (control and treatment) samples. Movies were assembled using Imaris (Oxford Instruments), Premiere Pro (Adobe), and After Effects (Adobe). 


\section{Statistical analyses}

For each zebrafish experiment, data from two or more independent biological replicates with separate cohorts of embryos were analyzed for the main effects of treatment.

For analyses of hemorrhage in rhoad ${ }^{R 17}$ mutant embryos, homogeneity across replicates was determined using the $G$-test of independence, and homogenous datasets (heterogeneity $G$ value $\geq 0.05)$ were combined for statistical analysis. Heterogeneous replicate datasets were analyzed separately and combined only when statistical analyses yielded identical results for each replicate dataset. Significant differences among treatments were determined using twotailed Fisher's exact tests on cumulative raw counts, with Bonferroni correction applied to multiple comparisons (alpha $=0.05)$.

For analyses of wild type versus rhoaa $a^{\Delta 3 /+}$ incross progeny, embryos with a specific phenotype were categorized (wild type, hemorrhaging, no circulation; wild type CtAs, reduced CtAs, overbranching CtAs) and counted. Homogeneity across replicates was determined using the $G$ test of independence, and homogenous datasets (heterogeneity G-value $\geq 0.05$ ) were combined for statistical analysis. Heterogeneous replicate datasets were analyzed separately and combined only when statistical analyses yielded identical results for each replicate dataset. Significant differences among treatments were determined using Fisher-Freeman-Halton exact tests of independence on raw counts (alpha $=0.05$ ).

For EC-specific transgenic Tomato or rhoaa isoform overexpression experiments and Rockout treatment experiments, significant phenotypic differences among groups or treatments across 
bioRxiv preprint doi: https://doi.org/10.1101/2021.03.27.437282; this version posted March 27, 2021. The copyright holder for this preprint

(which was not certified by peer review) is the author/funder, who has granted bioRxiv a license to display the preprint in perpetuity. It is made available under aCC-BY-NC-ND 4.0 International license.

biological replicates were determined using the Cochran-Mantel-Haenszel test for repeated $2 \times 2$

tests of independence (alpha $=0.05)$. 


\section{RESULTS}

\section{Zebrafish rhoaa mutants with distinct cranial vascular integrity and patterning defects}

We identified a novel mutant (y172) in a forward-genetic ENU mutagenesis screen for dominant or partially-dominant zebrafish mutants with intracranial hemorrhage (Figure S1A,B).

Genetic fine mapping and genome sequencing suggested that the causative defect in the $y 172$ mutant is a Glycine to Arginine substitution at amino acid 17 in the evolutionarily conserved GTP/GDP-binding domain ${ }^{73,74}$ of rhoaa, a zebrafish orthologue of mammalian RHOA (Figure 1A; Figure S1C-F). We therefore subsequently refer to $y 172$ mutants as rhoaa ${ }^{R 17}$ or R17 mutants. Whole-mount in situ hybridization analyses show that rhoaa is broadly expressed in wild type embryos at 26 hours post fertilization (hpf), with particularly strong expression in the head (Figure 1B), becoming almost exclusively expressed in the head by 48 hpf (Figure 1C,D). Starting at approximately $48 \mathrm{hpf}$, both R17/+ heterozygous and R17/R17 homozygous mutant embryos begin to exhibit incompletely penetrant cranial hemorrhage (Figure 1E-G,M) with variable expressivity ranging from small hematomas barely visible under a light microscope to large hemorrhages that fill one or more brain ventricles (Figure S1B). To determine if R17 mutants have altered blood vessel morphology or patterning, we crossed them to a $T g(k d r l: E G F P)^{s 843}, T g(g a t a 1: D s R e d)^{s d 2 T g}$ double transgenic reporter line with EGFP-labeled endothelial cells (ECs) and DsRed-labeled primitive blood cells ${ }^{48,49}$. By 52 hpf, both R17/+ and R17/R17 embryos develop vasculogenic primary cranial blood vessels, including the lateral dorsal aortae (LDA) and primordial hindbrain channels (PHBC), as well as angiogenic cranial central arteries (CtA), although CtA growth is sometimes slightly delayed in R17/R17 animals 
(Figure 1I-L) $^{75}$. R17/+ and R17/R17 embryos also exhibit normal development of trunk blood vessels, including the dorsal aorta (DA), cardinal vein (CV), and intersegmental vessels (ISVs) at 52 hpf (Figure S2A-C).

The rhoaa gene is one of five highly-conserved zebrafish RHOA orthologues (Figure S3A,B) exhibiting highly overlapping developmental expression patterns (Figure S3C-H). The strong possibility of compensation by other zebrafish rhoa orthologues and the dominant nature of the R17 mutation suggest that R17 may be acting as either a dominant negative (DN) or constitutively active (CA) allele of rhoaa. In support of this hypothesis, previous studies have shown that mutating the RHOA GTP/GDP-binding domain yields both DN and CA RHOA alleles. For example, RHOA ${ }^{\mathrm{V} 14}$ is a CA version of human RHOA that is unable to hydrolyze GTP, while $\mathrm{RHOA}^{\mathrm{N} 19}$ is a DN version of human RHOA that preferentially remains bound to GDP 73,76 (Figure 1A). To help further explore the effects of and validate the R17 mutation, we used CRISPR/Cas9 mutagenesis ${ }^{63}$ to generate an additional zebrafish mutant with a small, in-frame 3-base pair deletion and amino acid substitution in the Rhoaa GTP/GDP-binding domain, (designated $r a_{a a^{\Delta 3}}$ or $\Delta 3$; Figure $\left.1 \mathrm{~A}\right)$. We found that the R17 and $\Delta 3$ mutants exhibit partially overlapping but distinct phenotypes (Figure 1, Figure S2).

Like R17/+ heterozygotes, $\Delta 3 /+$ heterozygous embryos exhibit wild type morphology with incompletely penetrant cranial hemorrhage beginning at $48 \mathrm{hpf}$ (Figure 10,V) and normal cranial and trunk blood vessel patterning at $52 \mathrm{hpf}$ (Figure 1R, Figure S2E). However, unlike R17/R17 animals, most $\Delta 3 / \Delta 3$ homozygotes lack blood circulation and exhibit heart edema, despite having a heartbeat (Movie S1; Figure 1P,V). They also have smaller heads with atypical morphology (Figure 1P). Although the vasculogenic primary cranial vessels (LDA, PHBC) are 
normally patterned in $\Delta 3 / \Delta 3$ homozygous mutants, they exhibit ectopic growth and branching of the angiogenic central arteries (CtA) and, as might perhaps be expected from the lack of circulation, their vessels are poorly dilated (Figure 1S,U,W). Overall trunk blood vessel patterning is normal in $52 \mathrm{hpf} \Delta 3 / \Delta 3$ homozygotes, although these vessels (especially the ISVs) also fail to fully dilate (Figure S2F). Together, these data suggest that while R17/+ and $\Delta 3 /+$ heterozygotes both exhibit impaired cranial vascular integrity, $\Delta 3 / \Delta 3$ homozygotes have additional vascular defects not found in R17/R17 homozygotes.

The difference between R17/R17 and $\Delta 3 / \Delta 3$ homozygotes does not appear to be the result of differences in the relative amounts of Rhoa proteins, since both show very similar (approximately 50\%) reductions in total Rhoa, as measured by western blotting of wholeembryo zebrafish protein lysates collected at $50 \mathrm{hpf}$ (Figure S4). These results suggest that the Rhoaa $^{\mathrm{R} 17}$ and $\mathrm{Rhoaa}^{\Delta 3}$ proteins may instead possess distinct activities.

\section{Rhoaa alleles have differential effects on adherens junctions in cultured primary ECs in vitro}

Vascular permeability is modulated primarily by the integrity of EC-EC adherens and tight junction complexes ${ }^{2}$. Adherens junctions consist of transmembrane cadherins, such as Cdh5/VE-Cadherin. Previous studies using primary human umbilical vein ECs (HUVEC) in vitro have shown that increased RHOA activity disrupts adherens junctions (Haidari et al., 2011; Wojciak-Stothard et al., 2002), while dominant-negative suppression of RHOA signaling does not alter the distribution of adherens junction proteins in unstimulated cells (Wójciak-Stothard et al., 2001). To examine the effects of expressing different mutant rhoaa proteins on EC-EC junctions in vitro, we used lentiviral transduction to generate stable HUVEC lines expressing 


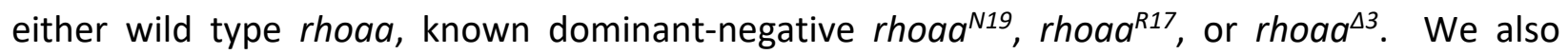
attempted to generate stable HUVEC lines expressing known constitutively-active rhoaa ${ }^{\mathrm{V} 14}$, but cells transduced with this allele are not viable (data not shown). We examined adherens junction morphology in confluent monolayers of stable rhoaa isoform-expressing HUVEC lines using immunohistochemical staining with an anti-Cdh5 antibody (Figure 2).

Compared to "empty-vector" transduced controls (Figure 2A,F), HUVEC expressing WT rhoaa exhibit jagged, highly discontinuous junctional Cdh5 staining (Figure 2B,G), as previously reported for ECs with excess RHOA pathway activation (Pronk et al., 2017). HUVEC expressing rhoaa $^{R 17}$ also exhibit jagged junctional staining (Figure $2 \mathrm{C}, \mathrm{H}$ ), although in this case, the staining is much less discontinuous than in HUVEC expressing WT rhoaa. In contrast, HUVEC expressing either the known dominant-negative $\operatorname{rhoaa}^{\mathrm{N19}}$ (Figure 2D,I) or rhoaa ${ }^{\Delta 3}$ (Figure 2E,J) exhibit thin, mostly continuous junctional Cdh5 staining.

Together these results suggest that the $\mathrm{Rhoaa}^{\mathrm{R} 17}$ protein may have reduced or weak dominantnegative activity, while Rhoaa ${ }^{\Delta 3}$ likely acts as a strong dominant-negative allele of Rhoaa.

Vascular defects are associated with either increased and decreased Rhoaa levels or activity in the zebrafish vascular endothelium in vivo

To further explore the in vivo consequences of increased or decreased Rhoa activity specifically in the vascular endothelium, we generated a series of $\mathrm{Tg}$ (UAS:Tomato-2A-rhoaa) transgenic zebrafish co-expressing Tomato fluorescent protein together with either wild type rhoaa, the rhoaa $^{R 17}$ or rhoad ${ }^{\Delta 3}$ mutant isoforms, or known dominant-negative or constitutively-active rhoaa isoforms (DN rhoaa ${ }^{N 19}$ or $C A r^{2 h o a a^{14}}$ ) under Gal4 control. We crossed these germline 
UAS:Tomato-2A-rhoaa transgenics to Tg(egfl7:GAL4ff); Tg(UAS:EGFP) double-transgenic zebrafish with vascular EC-specific Gal4 and EGFP reporter expression (Figure 3A).

EC-specific expression of Tomato alone has no effect on vascular integrity or development in zebrafish embryos (Figure S5). Compared to their control Tomato-negative siblings (Figure 3B$\mathrm{E}, \mathrm{N}, \mathrm{Q}-\mathrm{T})$, embryos expressing ectopic WT rhoaa in ECs exhibit incompletely penetrant cranial hemorrhage (Figure $3 \mathrm{~F}, \mathrm{Q}$ ), lack of circulation (Figure $3 \mathrm{R}$ ), reduced growth of cranial CtAs (Figure 3G,H,S), impaired growth of trunk ISVs (Figure 3I,O,T), and vessel dilation defects (Figure 3G-I,0). Embryos expressing CA rhoaav14 in ECS also sometimes exhibit cranial hemorrhage (Figure 3Q) and have much more penetrant and severe circulation, CtA/ISV angiogenesis, and blood vessel dilation defects (Figure 3K-M,P,R-T, Movie S2) than either WTrhoaa expressing animals or their control Tomato-negative siblings (Figure 3R-T, Figure S6K-

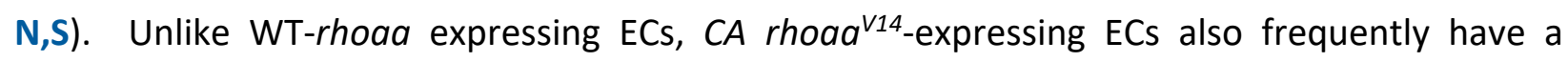
rounded, punctate morphology and/or appear fragmented, particularly in rhoaa ${ }^{\mathrm{V} 14}$-expressing cranial ECs (Figure 3K-M, Movie S3). Combined, these results suggest that increased Rhoaa dosage and/or activity in zebrafish ECs produces both vascular integrity and vascular growth defects, with high levels of Rhoa activation (in CA rhoaa ${ }^{\mathrm{V} 14}$-expressing ECs) inducing rounded, punctate EC morphology and/or EC fragmentation.

To study the consequences of decreased Rhoaa activity the vascular endothelium in vivo, we examined transgenic zebrafish embryos with EC-specific expression of known dominantnegative $D N$ rhoaa $^{N 19}$. Compared to Tomato-negative sibling controls (Figure $4 \mathrm{~A}-\mathrm{D}, \mathrm{Q}, \mathrm{U}, \mathrm{V}$ ) embryos expressing $D N$ rhoaa ${ }^{N 19}$ in ECs exhibit severe cranial hemorrhage (Figure $4 \mathrm{E}, \mathrm{U}$ ) and reduced CtA vessel growth at $52 \mathrm{hpf}$ (Figure 4F,G,V). Unlike cranial CtAs expressing EC-specific 
$D N$ rhoaa $^{N 19}$, most trunk ISVs expressing this isoform grow well and successfully reach the DLAV by $52 \mathrm{hpf}$, although a subset of ISVs are abnormally dilated and/or detach from the DA, CV, or DLAV (Figure 4H,R, Movie S4). Like embryos expressing $D N r_{h o a a^{N 19}}$ in the vascular endothelium, compared to Tomato-negative sibling controls (Figure 4U,V, Figure S7K-N,S), embryos with EC-specific rhoaa ${ }^{\Delta 3}$ expression exhibit severe cranial hemorrhage, reduced CtA vessel growth, and abnormally dilated and/or detached ISVs (Figure 4I-L,S,U,V), supporting the idea that Rhoaa ${ }^{\Delta 3}$ is acting as a dominant-negative Rhoaa protein.

In contrast, compared to Tomato-negative sibling controls (Figure $4 \mathrm{U}, \mathrm{V}$, Figure S7U-X, $\mathrm{C}^{\prime}$ ) zebrafish embryos with transgene-driven EC-specific expression of the Rhoaa ${ }^{\mathrm{R} 17}$ protein show relatively low levels of cranial hemorrhage and no reduction in CtA growth (Figure $4 \mathrm{M}-\mathrm{O}, \mathrm{U}, \mathrm{V}$ ). A small subset of these embryos do exhibit abnormally dilated and/or detached trunk ISVs (Figure 4P,T), although this phenotype is not as severe or penetrant as seen in DN rhoaa ${ }^{N 19}$ (Figure $4 \mathrm{H}, \mathrm{R}$ ) or $D N$ rhoag $^{\Delta 3}$ (Figure $4 \mathrm{~L}, \mathrm{~S}$ ) expressing animals. These results suggest that Rhoaa $^{\mathrm{R} 17}$ may be a very weak, dominant negative isoform of Rhoaa compared to DN Rhoaa ${ }^{\mathrm{N} 19}$ or Rhoaa ${ }^{\Delta 3}$.

\section{Rock function is required for cranial angiogenesis and to maintain vascular integrity in vivo}

The Rho-associated protein kinases ROCK1 and ROCK2 are some of the best characterized RHOA effectors ${ }^{11}$. Whole-mount in situ hybridization analyses of the zebrafish ROCK orthologues rock1 and rock2a revealed that their expression is enriched in the head at $52 \mathrm{hpf}$, in nearly identical patterns to rhoaa (Figure 5A-D), making them attractive candidates to mediate RHOA signaling in regulating cranial blood vessel growth and integrity. To determine 
whether loss of Rock1/2 activity results in comparable vascular defects to rhoaa ${ }^{\Delta / \Delta 3}$ mutants or animals with EC-specific expression of rhoad $^{\Delta 3}$, we treated zebrafish from 24 - 52 hpf with Rockout, a cell-permeable pharmacological inhibitor of Rock1/2 kinase activity previously used in zebrafish $45,77,78$. This treatment interval was selected to bypass earlier gastrulation defects caused by Rock inhibition ${ }^{79}$. Rockout-treated animals exhibit dose-dependent, highly penetrant cranial hemorrhage and CtA growth defects at $52 \mathrm{hpf}$ (Figure $5 \mathrm{E}-\mathrm{N}$ ). 


\section{DISCUSSION}

In this study, we have examined the function of the small, monomeric GTPase Rhoa in regulating vascular development and integrity in vivo. We used zebrafish RHOA-ortholog (rhoaa) mutants, transgenic embryos expressing wild type, dominant-negative, or constitutively active forms of rhoaa in ECs, and a pharmacologic inhibitor of the Rhoa effectors Rock1/2 to study the in vivo developmental consequences of Rhoa gain- and loss-of-function in the vascular endothelium. Our findings document roles for Rhoa function in vascular integrity, developmental angiogenesis, and vascular morphogenesis in vivo.

\section{Identification and characterization of novel zebrafish mutant alleles of Rhoa}

We isolated and characterized two mutants carrying novel alleles of the zebrafish RHOAorthologue rhoaa (rhoaa ${ }^{3}$ and rhoaa ${ }^{R 17}$; Figure 1). Both mutants display partially dominant cranial hemorrhage phenotypes as heterozygotes. However, while rhoaa ${ }^{3 / 33}$ homozygotes fail to develop circulation and exhibit both cranial vascular patterning and morphological defects, $r a_{a}^{R 17 / R 17}$ homozygotes exhibit wild type circulation, vascular patterning, and morphology. Both mutants have comparable levels of total Rhoa protein, suggesting that their phenotypic differences reflect differences in Rhoaa isoform activities, not protein levels. This idea is supported by the differential phenotypes of ECs expressing rhoaa ${ }^{R 17}$ or rhoaa ${ }^{3}$ both in vitro in primary human ECs (Figure 2) and in vivo in zebrafish embryos (Figure 4).

Human umbilical vein endothelial cells (HUVEC) expressing either the known dominantnegative (DN) $r$ oad ${ }^{N 19}$ or rhoaa $^{3}$ show essentially identical adherens junction morphology, while HUVEC expressing rhoad ${ }^{R 17}$ show an intermediate phenotype between cells expressing 
wild type rhoaa and cells expressing $D N$ rhoaa $^{N 19}$ or rhoaa ${ }^{33}$ (Figure 2). Similarly, zebrafish with transgene-driven EC-specific expression of either DN rhoad ${ }^{N 19}$ or rhoaa ${ }^{3}$ exhibit severe cranial hemorrhage, a strong reduction in angiogenic cranial vessel growth, and dilation/detachment of trunk vessels. rhoa $a^{R 17}$-expressing zebrafish embryos display far less penetrant cranial hemorrhage and trunk vessel dilation/detachment phenotypes, and no reduction in cranial vessel growth (Figure 4). In contrast to the results with these other mutants, the punctate EC morphologies and EC fragmentation observed in the known constitutive-active rhoaa ${ }^{\mathrm{V} 14}$ mutant suggest that this mutant may be incompatible with endothelial cell viability when expressed in either HUVEC in vitro or in zebrafish endothelial cells in vivo (Figure 3). Together, these findings

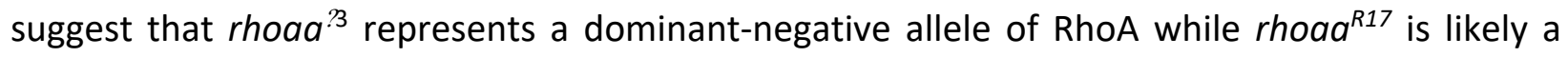
weak dominant-negative allele.

\section{Rhoa and vascular integrity}

Previous in vitro studies have implicated RHOA/ROCK function in EC barrier disruption in response to permeability inducing factors such as vascular endothelial growth factor, thrombin, histamine, and tumor necrosis factor alpha $15,20,36,80-87$. RHOA/ROCK have also been shown to mediate EC barrier enhancement in response to angiopoietin 1 and sphingosine-1-phosphate ${ }^{22-}$ $24,88,89$. Our zebrafish studies suggest that the in vivo developing cranial vasculature is exquisitely sensitive to either increased or decreased Rhoa dosage or activity (Figure 6). Cranial hemorrhage occurs in heterozygotes of both the stronger dominant-negative rhoaa ${ }^{33}$ mutant and the weaker rhoad ${ }^{R 17}$ mutant (Figure 1), as well as in transgenic embryos with EC-specific expression of rhoaa $^{33}$ or rhoad ${ }^{R 17}$ (Figure 4). Embryos treated with the ROCK1/2 inhibitor 
Rockout (Figure 5) also hemorrhage, indicating that decreased RHOA/ROCK function leads to disruption of vascular integrity in vivo. Transgene-driven expression of ectopic wild type rhoaa in ECs also leads to cranial hemorrhage in zebrafish (Figure 3) and causes adherens junction disruption in HUVEC (Figure 2), suggesting that increased RHOA/ROCK function can also disrupt vascular integrity. Our combined results therefore suggest that a proper level of Rhoa/Rock signaling is required to maintain cranial vascular integrity in vivo under normal physiologic conditions, with either too much or too little resulting in disruption of barrier function (Figure 6).

It remains unclear why the barrier-disrupting effects of increased or decreased RHOA/ROCK activity are restricted to the cranial vessels of zebrafish. In rhoaa mutants, EC-specific rhoaa transgene-expressing embryos, and Rock-inhibitor treated embryos, hemorrhage is observed in cranial, but not trunk vasculature beginning at $48 \mathrm{hpf}$. The trunk vasculature, does, however, exhibit other phenotypes such as impaired angiogenesis, vessel detachment, or vessel dilation (eg, Figure 4R-T). It is not clear what intrinsic molecular or physiological properties make the cranial EC barrier more sensitive to increased or decreased Rhoa activity and/or more prone to rupture than the trunk EC barrier at this developmental time point. Zebrafish embryos undergo a significant increase in peak blood pressure between $40 \mathrm{hpf}$ and $60 \mathrm{hpf}{ }^{90}$, a time when cranial vessels are still undergoing significant growth and remodeling, which could impact junctional adhesion. Trunk vessel angiogenesis (ISV growth to the DLAV) is largely complete by $40 \mathrm{hpf}^{75}$, so the differential sensitivity of cranial versus trunk vessels at this time could reflect differences in the timing of their growth and development. Alternatively, there may be intrinsic differences 
in the molecular and genetic profiles of cranial versus trunk ECs contributing to their differential sensitivity to gain or loss of Rhoa/Rock activity.

Interestingly, rhoaa mutants (rhoaa ${ }^{R 17 /+}$, rhoaa ${ }^{R 17 / R 17}$, and rhoaa ${ }^{3 /+}$ embryos) typically exhibit recovery of the EC barrier and clearance of pooled erythrocytes from the brain by 3 dpf (data not shown). This is approximately when EC barrier-protective pericytes are recruited to cranial blood vessels and the EC barrier begins to adopt a less permeable blood brain barrier (BBB) identity, characterized by increased numbers of junction proteins and reduced non-specific transcytosis events ${ }^{91-95}$. Developing cranial vessels may therefore be particularly sensitive to Rhoa levels only prior to pericyte recruitment and/or BBB development.

\section{Rhoa and developmental angiogenesis}

Previous in vitro studies have shown that RHOA/ROCK is required for EC migration, proliferation, and angiogenic tip cell protrusion in response to exogenous stimulation by S1P, VEGF, and/or flow $19,40,80$. We find that strong suppression of Rhoa function in transgenic zebrafish with EC-specific expression of dominant-negative rhoaa ${ }^{N 19}$ or rhoaa ${ }^{33}$ or animals treated with ROCK1/2 inhibitor results in dramatically reduced cranial vessel angiogenesis, but has a limited effect on trunk vessel angiogenesis. The presumably weaker suppression of Rhoa function in $r{ } a a^{R 17}$ or rhoaa $^{33}$ mutants, while sufficient to disrupt cranial vascular integrity (as is evident from the cranial hemorrhage in these embryos), is insufficient to disrupt cranial vessel angiogenesis. Interestingly, reduced cranial vessel angiogenesis is also noted in transgenic embryos expressing ectopic wild type rhoaa or constitutively-active rhoaa ${ }^{\mathrm{V} 14}$ in ECs (Figure 3), although rhoaa ${ }^{V 14}$ expression might be causing EC death independently from its 
inhibitory effects on angiogenesis (note again our inability to maintain stable lines of rhoaa ${ }^{\text {v14 }}$ expressing HUVEC and the punctate, sometimes fragmented appearance of rhoaa ${ }^{\text {14 }}$-expressing ECs in transgenic zebrafish). Together, these results suggest that, as for vascular integrity, cranial developmental angiogenesis is disrupted by too little or too much Rhoa activity.

The reasons for the more severe angiogenic defects in cranial vs. trunk vessels are unclear, although the later growth of cranial vessels compared to trunk intersegmental vessels could be a factor. Alternatively, the differential sensitivity of cranial vessels to angiogenesis defects in response to altered $\mathrm{RHOA}$ /ROCK could reflect intrinsic molecular differences between ECs in these different vascular beds.

\section{Rhoa and vascular morphogenesis}

Zebrafish with altered vascular endothelial Rhoa function also exhibit defects in vascular morphogenesis and blood vessel dilation. A previous report showed that HUVEC with excess RHOA activity have impaired vacuole formation and fail to form "lumens" in in vitro cell culture tubulogenesis assays ${ }^{14}$. EC-specific Rhoa knockout mice exhibit increased blood vessel lumen diameter, and EC-specific Rhoa knockout can partially rescue lumen formation in Ras interacting protein 1 (Rasip1) knockout mouse embryos, which normally exhibit increased RHOA/ROCK activity ${ }^{39}$, supporting the idea that RHOA functions to inhibit or limit vessel lumenogenesis in vivo.

Our results are generally in line with this previously described presumptive activity for RHOA. In transgenic zebrafish with EC-specific expression of ectopic wild type rhoaa, vessel segments strongly expressing the transgene (as indicated by robust Tomato reporter fluorescence) are 
frequently narrow and undilated. In contrast, vessel segments in transgenic zebrafish with strong EC-specific expression of dominant-negative rhoaa ${ }^{N 19}$, rhoaa ${ }^{3}$, or rhoad ${ }^{R 17}$ isoforms often appear excessively dilated compared to vessel segments in the same animals with less, or no transgene expression. The range of vessel dilation phenotypes observed in these individual "mosaics" strongly suggests that they are not due to reduced cardiac function and/or impaired circulation. Interestingly, zebrafish embryonic trunk vessels expressing the dominant-negative isoforms of rhoaa also undergo sporadic detachment (Figure 4). This phenotype is not accompanied by hemorrhage, suggesting that it is not simply the result of generalized ECbarrier breakdown. Future studies will be needed to define the molecular basis for this phenotype.

\section{Rhoa function in the vasculature}

Together, our findings highlight roles for Rhoa function in vascular integrity, angiogenesis, and vascular morphogenesis during development (Figure 6). Too much or too little Rhoa function impairs cranial vascular integrity and cranial developmental angiogenesis. Cranial vascular integrity appears to be more sensitive to altered Rhoa gene dosage or activity than cranial angiogenesis, which apparently tolerates more substantial deviations from normal Rhoa levels or activity. Our zebrafish results largely support previous reports that RHOA functions to inhibit or limit vessel lumenogenesis. Suppressing Rhoa function in zebrafish endothelium leads to vessel dilation, while excess EC Rhoa results in undilated vessels. In addition to providing important new information on the in vivo roles of Rhoa in the vascular endothelium, our study highlights the power of the zebrafish as a model for genetic and experimental dissection of vascular biology in an intact animal. 


\section{Acknowledgements:}

The authors would like to thank members of the Weinstein laboratory for their critical comments on this manuscript. Schematics of larval zebrafish were created with BioRender.com.

\section{Sources of Funding:}

This work was supported by the intramural program of the Eunice Kennedy Shriver National Institute of Child Health and Human Development, National Institutes of Health (ZIA-HD001011 and ZIA-HD008915, to BMW).

Disclosures: The authors declare no competing interests or disclosures.

\section{Supplemental Materials:}

Figures S1-S7

Table S1

Movies S1-S4

Correspondence: Brant M. Weinstein, Division of Developmental Biology, Eunice Kennedy Shriver National Institute of Child Health and Human Development, National Institutes of Health, 6 Center Dr. Bethesda, MD 20892; e-mail: flyingfish2@nih.gov 


\section{REFERENCES}

1. Hu X, De Silva TM, Chen J, Faraci FM. Cerebral vascular disease and neurovascular injury in ischemic stroke. Circ Res. 2017;120:449-471

2. Radeva MY, Waschke J. Mind the gap: Mechanisms regulating the endothelial barrier. Acta Physiol (Oxf). 2018;222

3. Harris TJ, Tepass U. Adherens junctions: From molecules to morphogenesis. Nat Rev Mol Cell Biol. 2010;11:502-514

4. van Buul JD, Timmerman I. Small rho gtpase-mediated actin dynamics at endothelial adherens junctions. Small GTPases. 2016;7:21-31

5. Beckers CM, van Hinsbergh VW, van Nieuw Amerongen GP. Driving rho gtpase activity in endothelial cells regulates barrier integrity. Thromb Haemost. 2010;103:40-55

6. Wojciak-Stothard B, Ridley AJ. Rho gtpases and the regulation of endothelial permeability. Vascul Pharmacol. 2002;39:187-199

7. Barlow HR, Cleaver O. Building blood vessels-one rho gtpase at a time. Cells. 2019;8

8. Nobes C, Hall A. Regulation and function of the rho subfamily of small gtpases. Curr Opin Genet Dev. 1994;4:77-81

9. Amano $\mathrm{M}$, Chihara K, Kimura K, Fukata $\mathrm{Y}$, Nakamura N, Matsuura $\mathrm{Y}$, Kaibuchi K. Formation of actin stress fibers and focal adhesions enhanced by rho-kinase. Science. $1997 ; 275: 1308-1311$ 
10. Jaffe $A B$, Hall $A$. Rho gtpases: Biochemistry and biology. Annu Rev Cell Dev Biol. $2005 ; 21: 247-269$

11. Yao L, Romero MJ, Toque HA, Yang G, Caldwell RB, Caldwell RW. The role of rhoa/rho kinase pathway in endothelial dysfunction. J Cardiovasc Dis Res. 2010;1:165-170

12. Shih YP, Yuan SY, Lo SH. Down-regulation of dlc1 in endothelial cells compromises the angiogenesis process. Cancer Lett. 2017;398:46-51

13. El Atat O, Fakih A, El-Sibai M. Rhog activates rac1 through cdc42 leading to tube formation in vascular endothelial cells. Cells. 2019;8

14. Bayless KJ, Davis GE. The cdc42 and rac1 gtpases are required for capillary lumen formation in three-dimensional extracellular matrices. J Cell Sci. 2002;115:1123-1136

15. Bryan BA, Dennstedt E, Mitchell DC, Walshe TE, Noma K, Loureiro R, Saint-Geniez M, Campaigniac JP, Liao JK, D'Amore PA. Rhoa/rock signaling is essential for multiple aspects of vegf-mediated angiogenesis. FASEB J. 2010;24:3186-3195

16. Ridley AJ, Hall A. The small gtp-binding protein rho regulates the assembly of focal adhesions and actin stress fibers in response to growth factors. Cell. 1992;70:389-399

17. Soga N, Namba N, McAllister S, Cornelius L, Teitelbaum SL, Dowdy SF, Kawamura J, Hruska KA. Rho family gtpases regulate vegf-stimulated endothelial cell motility. Exp Cell Res. 2001;269:73-87 
18. Pronk MCA, van Bezu JSM, van Nieuw Amerongen GP, van Hinsbergh VWM, Hordijk PL. Rhoa, rhob and rhoc differentially regulate endothelial barrier function. Small GTPases. 2017:1-19

19. van Nieuw Amerongen GP, Koolwijk P, Versteilen A, van Hinsbergh VW. Involvement of rhoa/rho kinase signaling in vegf-induced endothelial cell migration and angiogenesis in vitro. Arterioscler Thromb Vasc Biol. 2003;23:211-217

20. van Nieuw Amerongen GP, van Delft S, Vermeer MA, Collard JG, van Hinsbergh VW. Activation of rhoa by thrombin in endothelial hyperpermeability: Role of rho kinase and protein tyrosine kinases. Circ Res. 2000;87:335-340

21. Oldenburg J, de Rooij J. Mechanical control of the endothelial barrier. Cell Tissue Res. $2014 ; 355: 545-555$

22. Gavard J, Patel V, Gutkind JS. Angiopoietin-1 prevents vegf-induced endothelial permeability by sequestering src through mdia. Dev Cell. 2008;14:25-36

23. $\mathrm{Xu} \mathrm{M}$, Waters $\mathrm{CL}, \mathrm{Hu} \mathrm{C}$, Wysolmerski RB, Vincent PA, Minnear FL. Sphingosine 1phosphate rapidly increases endothelial barrier function independently of ve-cadherin but requires cell spreading and rho kinase. Am J Physiol Cell Physiol. 2007;293:C13091318

24. Zhang XE, Adderley SP, Breslin JW. Activation of rhoa, but not rac1, mediates early stages of s1p-induced endothelial barrier enhancement. PLoS One. 2016;11:e0155490

25. Pedersen E, Brakebusch C. Rho gtpase function in development: How in vivo models change our view. Exp Cell Res. 2012;318:1779-1787 
26. Kaunas R, Nguyen P, Usami S, Chien S. Cooperative effects of rho and mechanical stretch on stress fiber organization. Proc Natl Acad Sci U S A. 2005;102:15895-15900

27. Shikata Y, Rios A, Kawkitinarong K, DePaola N, Garcia JG, Birukov KG. Differential effects of shear stress and cyclic stretch on focal adhesion remodeling, site-specific fak phosphorylation, and small gtpases in human lung endothelial cells. Exp Cell Res. 2005;304:40-49

28. Yamazaki Y, Kanekiyo T. Blood-brain barrier dysfunction and the pathogenesis of alzheimer's disease. Int J Mol Sci. 2017;18

29. Zafar A, Quadri SA, Farooqui M, Ikram A, Robinson M, Hart BL, Mabray MC, Vigil C, Tang AT, Kahn ML, Yonas H, Lawton MT, Kim H, Morrison L. Familial cerebral cavernous malformations. Stroke. 2019;50:1294-1301

30. Stockton RA, Shenkar R, Awad IA, Ginsberg MH. Cerebral cavernous malformations proteins inhibit rho kinase to stabilize vascular integrity. J Exp Med. 2010;207:881-896

31. Borikova AL, Dibble CF, Sciaky N, Welch CM, Abell AN, Bencharit S, Johnson GL. Rho kinase inhibition rescues the endothelial cell cerebral cavernous malformation phenotype. J Biol Chem. 2010;285:11760-11764

32. Whitehead KJ, Chan AC, Navankasattusas S, Koh W, London NR, Ling J, Mayo AH, Drakos SG, Jones CA, Zhu W, Marchuk DA, Davis GE, Li DY. The cerebral cavernous malformation signaling pathway promotes vascular integrity via rho gtpases. Nat Med. 2009;15:177- 
33. McDonald DA, Shi C, Shenkar R, Stockton RA, Liu F, Ginsberg MH, Marchuk DA, Awad IA. Fasudil decreases lesion burden in a murine model of cerebral cavernous malformation disease. Stroke. 2012;43:571-574

34. Shenkar R, Shi C, Austin C, Moore T, Lightle R, Cao Y, Zhang L, Wu M, Zeineddine HA, Girard R, McDonald DA, Rorrer A, Gallione C, Pytel P, Liao JK, Marchuk DA, Awad IA. Rhoa kinase inhibition with fasudil versus simvastatin in murine models of cerebral cavernous malformations. Stroke. 2017;48:187-194

35. Shenkar R, Peiper A, Pardo H, Moore T, Lightle R, Girard R, Hobson N, Polster SP, Koskimaki J, Zhang D, Lyne SB, Cao Y, Chaudagar K, Saadat L, Gallione C, Pytel P, Liao JK, Marchuk D, Awad IA. Rho kinase inhibition blunts lesion development and hemorrhage in murine models of aggressive pdcd10/ccm3 disease. Stroke. 2019;50:738-744

36. Mikelis CM, Simaan M, Ando K, Fukuhara S, Sakurai A, Amornphimoltham P, Masedunskas A, Weigert R, Chavakis T, Adams RH, Offermanns S, Mochizuki N, Zheng Y, Gutkind JS. Rhoa and rock mediate histamine-induced vascular leakage and anaphylactic shock. Nat Commun. 2015;6:6725

37. Hoang MV, Whelan MC, Senger DR. Rho activity critically and selectively regulates endothelial cell organization during angiogenesis. Proc Natl Acad Sci U $S$ A. 2004;101:1874-1879

38. Park HJ, Kong D, Iruela-Arispe L, Begley U, Tang D, Galper JB. 3-hydroxy-3-methylglutaryl coenzyme a reductase inhibitors interfere with angiogenesis by inhibiting the geranylgeranylation of rhoa. Circ Res. 2002;91:143-150 
39. Barry DM, Koo Y, Norden PR, Wylie LA, Xu K, Wichaidit C, Azizoglu DB, Zheng Y, Cobb MH, Davis GE, Cleaver O. Rasip1-mediated rho gtpase signaling regulates blood vessel tubulogenesis via nonmuscle myosin ii. Circ Res. 2016;119:810-826

40. Zahra FT, Sajib MS, Ichiyama Y, Akwii RG, Tullar PE, Cobos C, Minchew SA, Doci CL, Zheng Y, Kubota Y, Gutkind JS, Mikelis CM. Endothelial rhoa gtpase is essential for in vitro endothelial functions but dispensable for physiological in vivo angiogenesis. Sci Rep. 2019;9:11666

41. Gore AV, Monzo K, Cha YR, Pan W, Weinstein BM. Vascular development in the zebrafish. Cold Spring Harb Perspect Med. 2012;2:a006684

42. Butler MG, Gore AV, Weinstein BM. Zebrafish as a model for hemorrhagic stroke. Methods Cell Biol. 2011;105:137-161

43. Stratman AN, Weinstein BM. Assessment of vascular patterning in the zebrafish. Methods Mol Biol. 2021;2206:205-222

44. Kimmel CB, Ballard WW, Kimmel SR, Ullmann B, Schilling TF. Stages of embryonic development of the zebrafish. Dev Dyn. 1995;203:253-310

45. Yarrow JC, Totsukawa G, Charras GT, Mitchison TJ. Screening for cell migration inhibitors via automated microscopy reveals a rho-kinase inhibitor. Chem Biol. 2005;12:385-395

46. Prince VE, Moens CB, Kimmel CB, Ho RK. Zebrafish hox genes: Expression in the hindbrain region of wild-type and mutants of the segmentation gene, valentino. Development. 1998;125:393-406 
47. Thisse C, Thisse B, Schilling TF, Postlethwait JH. Structure of the zebrafish snail1 gene and its expression in wild-type, spadetail and no tail mutant embryos. Development. 1993;119:1203-1215

48. Traver D, Paw BH, Poss KD, Penberthy WT, Lin S, Zon LI. Transplantation and in vivo imaging of multilineage engraftment in zebrafish bloodless mutants. Nat Immunol. $2003 ; 4: 1238-1246$

49. Jin SW, Beis D, Mitchell T, Chen JN, Stainier DY. Cellular and molecular analyses of vascular tube and lumen formation in zebrafish. Development. 2005;132:5199-5209

50. Lawson ND, Weinstein BM. In vivo imaging of embryonic vascular development using transgenic zebrafish. Dev Biol. 2002;248:307-318

51. Venero Galanternik M, Castranova D, Gore AV, Blewett NH, Jung HM, Stratman AN, Kirby MR, Iben J, Miller MF, Kawakami K, Maraia RJ, Weinstein BM. A novel perivascular cell population in the zebrafish brain. Elife. 2017;6

52. Kawakami K, Abe G, Asada T, Asakawa K, Fukuda R, Ito A, Lal P, Mouri N, Muto A, Suster ML, Takakubo H, Urasaki A, Wada H, Yoshida M. Ztrap: Zebrafish gene trap and enhancer trap database. BMC Dev Biol. 2010;10:105

53. Zhang Y, Werling U, Edelmann W. Slice: A novel bacterial cell extract-based DNA cloning method. Nucleic Acids Res. 2012;40:e55

54. Kim JH, Lee SR, Li LH, Park HJ, Park JH, Lee KY, Kim MK, Shin BA, Choi SY. High cleavage efficiency of a 2 a peptide derived from porcine teschovirus-1 in human cell lines, zebrafish and mice. PLoS One. 2011;6:e18556 
55. Provost E, Rhee J, Leach SD. Viral 2a peptides allow expression of multiple proteins from a single orf in transgenic zebrafish embryos. Genesis. 2007;45:625-629

56. Yokogawa T, Hannan MC, Burgess HA. The dorsal raphe modulates sensory responsiveness during arousal in zebrafish. J Neurosci. 2012;32:15205-15215

57. Horstick EJ, Jordan DC, Bergeron SA, Tabor KM, Serpe M, Feldman B, Burgess HA. Increased functional protein expression using nucleotide sequence features enriched in highly expressed genes in zebrafish. Nucleic Acids Res. 2015;43:e48

58. Lawson ND, Mugford JW, Diamond BA, Weinstein BM. Phospholipase c gamma-1 is required downstream of vascular endothelial growth factor during arterial development. Genes Dev. 2003;17:1346-1351

59. Neff MM, Turk E, Kalishman M. Web-based primer design for single nucleotide polymorphism analysis. Trends Genet. 2002;18:613-615

60. Neff MM, Neff JD, Chory J, Pepper AE. Dcaps, a simple technique for the genetic analysis of single nucleotide polymorphisms: Experimental applications in arabidopsis thaliana genetics. Plant J. 1998;14:387-392

61. Michaels SD, Amasino RM. A robust method for detecting single-nucleotide changes as polymorphic markers by pcr. Plant J. 1998;14:381-385

62. Meeker ND, Hutchinson SA, Ho L, Trede NS. Method for isolation of pcr-ready genomic DNA from zebrafish tissues. Biotechniques. 2007;43:610, 612, 614 
63. Gagnon JA, Valen E, Thyme SB, Huang P, Akhmetova L, Pauli A, Montague TG, Zimmerman S, Richter C, Schier AF. Efficient mutagenesis by cas9 protein-mediated oligonucleotide insertion and large-scale assessment of single-guide rnas. PLoS One. 2014;9:e98186

64. Labun K, Montague TG, Krause M, Torres Cleuren YN, Tjeldnes H, Valen E. Chopchop v3: Expanding the crispr web toolbox beyond genome editing. Nucleic Acids Res. 2019;47:W171-W174

65. Sood R, Carrington B, Bishop K, Jones M, Rissone A, Candotti F, Chandrasekharappa SC, Liu P. Efficient methods for targeted mutagenesis in zebrafish using zinc-finger nucleases: Data from targeting of nine genes using compozr or coda zfns. PLoS One. 2013;8:e57239

66. Stratman AN, Burns MC, Farrelly OM, Davis AE, Li W, Pham VN, Castranova D, Yano JJ, Goddard LM, Nguyen O, Galanternik MV, Bolan TJ, Kahn ML, Mukouyama YS, Weinstein BM. Chemokine mediated signalling within arteries promotes vascular smooth muscle cell recruitment. Commun Biol. 2020;3:734

67. Norrman K, Fischer Y, Bonnamy B, Wolfhagen Sand F, Ravassard P, Semb H. Quantitative comparison of constitutive promoters in human es cells. PLoS One. 2010;5:e12413

68. Liu JW, Pernod G, Dunoyer-Geindre S, Fish RJ, Yang H, Bounameaux H, Kruithof EK. Promoter dependence of transgene expression by lentivirus-transduced human bloodderived endothelial progenitor cells. Stem Cells. 2006;24:199-208 
69. Turner DL, Weintraub H. Expression of achaete-scute homolog 3 in xenopus embryos converts ectodermal cells to a neural fate. Genes Dev. 1994;8:1434-1447

70. Michaelson D, Silletti J, Murphy G, D'Eustachio P, Rush M, Philips MR. Differential localization of rho gtpases in live cells: Regulation by hypervariable regions and rhogdi binding. J Cell Biol. 2001;152:111-126

71. Michaelson D, Philips M. The use of gfp to localize rho gtpases in living cells. Methods Enzymol. 2006;406:296-315

72. Schindelin J, Arganda-Carreras I, Frise E, Kaynig V, Longair M, Pietzsch T, Preibisch S, Rueden C, Saalfeld S, Schmid B, Tinevez JY, White DJ, Hartenstein V, Eliceiri K, Tomancak P, Cardona A. Fiji: An open-source platform for biological-image analysis. Nat Methods. 2012;9:676-682

73. Ihara K, Muraguchi S, Kato M, Shimizu T, Shirakawa M, Kuroda S, Kaibuchi K, Hakoshima T. Crystal structure of human rhoa in a dominantly active form complexed with a gtp analogue. J Biol Chem. 1998;273:9656-9666

74. Wei Y, Zhang Y, Derewenda U, Liu X, Minor W, Nakamoto RK, Somlyo AV, Somlyo AP, Derewenda ZS. Crystal structure of rhoa-gdp and its functional implications. Nat Struct Biol. 1997;4:699-703

75. Isogai S, Horiguchi M, Weinstein BM. The vascular anatomy of the developing zebrafish: An atlas of embryonic and early larval development. Dev Biol. 2001;230:278-301 
76. Coso OA, Chiariello M, Yu JC, Teramoto H, Crespo P, Xu N, Miki T, Gutkind JS. The small gtp-binding proteins rac1 and cdc42 regulate the activity of the jnk/sapk signaling pathway. Cell. 1995;81:1137-1146

77. Weiser DC, Pyati UJ, Kimelman D. Gravin regulates mesodermal cell behavior changes required for axis elongation during zebrafish gastrulation. Genes Dev. 2007;21:15591571

78. Weiser DC, Kimelman D. Analysis of cell shape and polarity during zebrafish gastrulation. Methods Mol Biol. 2012;839:53-68

79. Lai SL, Chang CN, Wang PJ, Lee SJ. Rho mediates cytokinesis and epiboly via rock in zebrafish. Mol Reprod Dev. 2005;71:186-196

80. Song JW, Daubriac J, Tse JM, Bazou D, Munn LL. Rhoa mediates flow-induced endothelial sprouting in a 3-d tissue analogue of angiogenesis. Lab Chip. 2012;12:5000-5006

81. Shasby DM, Stevens T, Ries D, Moy AB, Kamath JM, Kamath AM, Shasby SS. Thrombin inhibits myosin light chain dephosphorylation in endothelial cells. Am J Physiol. 1997;272:L311-319

82. Essler M, Amano M, Kruse HJ, Kaibuchi K, Weber PC, Aepfelbacher M. Thrombin inactivates myosin light chain phosphatase via rho and its target rho kinase in human endothelial cells. J Biol Chem. 1998;273:21867-21874

83. Zeng $H$, Zhao D, Mukhopadhyay D. Kdr stimulates endothelial cell migration through heterotrimeric g protein gq/11-mediated activation of a small gtpase rhoa. J Biol Chem. $2002 ; 277: 46791-46798$ 
84. McKenzie JA, Ridley AJ. Roles of rho/rock and mlck in tnf-alpha-induced changes in endothelial morphology and permeability. J Cell Physiol. 2007;213:221-228

85. Huveneers S, de Rooij J. Mechanosensitive systems at the cadherin-f-actin interface. J Cell Sci. 2013;126:403-413

86. Huveneers S, Oldenburg J, Spanjaard E, van der Krogt G, Grigoriev I, Akhmanova A, Rehmann H, de Rooij J. Vinculin associates with endothelial ve-cadherin junctions to control force-dependent remodeling. J Cell Biol. 2012;196:641-652

87. Lisowska J, Rodel CJ, Manet S, Miroshnikova YA, Boyault C, Planus E, De Mets R, Lee HH, Destaing O, Mertani H, Boulday G, Tournier-Lasserve E, Balland M, Abdelilah-Seyfried S, Albiges-Rizo C, Faurobert E. The ccm1-ccm2 complex controls complementary functions of rock1 and rock2 that are required for endothelial integrity. J Cell Sci. 2018;131

88. Breslin JW, Zhang XE, Worthylake RA, Souza-Smith FM. Involvement of local lamellipodia in endothelial barrier function. PLoS One. 2015;10:e0117970

89. van Nieuw Amerongen GP, Beckers CM, Achekar ID, Zeeman S, Musters RJ, van Hinsbergh VW. Involvement of rho kinase in endothelial barrier maintenance. Arterioscler Thromb Vasc Biol. 2007;27:2332-2339

90. Yalcin HC, Amindari A, Butcher JT, Althani A, Yacoub M. Heart function and hemodynamic analysis for zebrafish embryos. Dev Dyn. 2017;246:868-880

91. Quinonez-Silvero C, Hubner K, Herzog W. Development of the brain vasculature and the blood-brain barrier in zebrafish. Dev Biol. 2020;457:181-190 
92. Eisa-Beygi S, Benslimane FM, El-Rass S, Prabhudesai S, Abdelrasoul MKA, Simpson PM, Yalcin HC, Burrows PE, Ramchandran R. Characterization of endothelial cilia distribution during cerebral-vascular development in zebrafish ( danio rerio). Arterioscler Thromb Vasc Biol. 2018;38:2806-2818

93. Wang Y, Pan L, Moens CB, Appel B. Notch3 establishes brain vascular integrity by regulating pericyte number. Development. 2014;141:307-317

94. Ando K, Fukuhara S, Izumi N, Nakajima H, Fukui H, Kelsh RN, Mochizuki N. Clarification of mural cell coverage of vascular endothelial cells by live imaging of zebrafish. Development. 2016;143:1328-1339

95. Hellstrom M, Kalen M, Lindahl P, Abramsson A, Betsholtz C. Role of pdgf-b and pdgfrbeta in recruitment of vascular smooth muscle cells and pericytes during embryonic blood vessel formation in the mouse. Development. 1999;126:3047-3055 


\section{FIGURES WITH FIGURE LEGENDS}

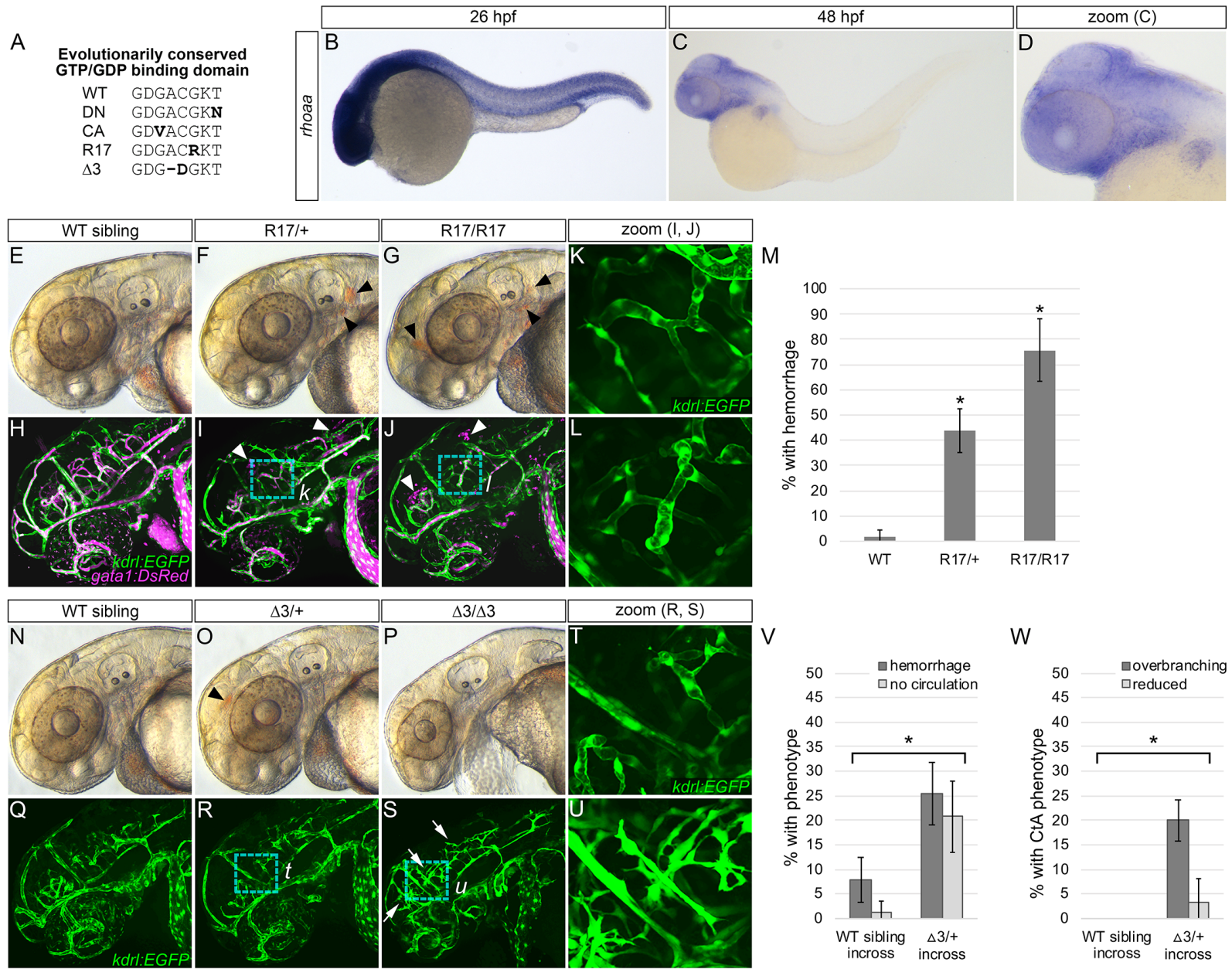

Figure 1. rhoaa mutant zebrafish embryos exhibit vascular integrity and patterning defects.

(A) Partial amino acid sequences of wild type (WT) Rhoaa, known dominant negative (DN) Rhoaa $^{\mathrm{N} 19}$, known constitutively active (CA) RhoaaV14, Rhoaa ${ }^{\mathrm{R} 17}$, Rhoaa ${ }^{3}$, and Rhoaa ${ }^{\Delta 6}$ GTP/GDPbinding domains. (B) Whole-mount in situ hybridization analysis of rhoaa gene expression in a 26 hours post fertilization (hpf) wild type (WT) zebrafish embryo shown in lateral view, anterior 
to left. (C) Whole-mount in situ hybridization analysis of rhoaa gene expression in a 48 hpf WT zebrafish embryo shown in lateral view, anterior to left. (D) Magnified view of head in C. (E-G) Stereoscope transmitted light images of $52 \mathrm{hpf} T g\left(k d r l: E G F P ;\right.$ gata1:DsRed); rhoaa ${ }^{R 17 /+}$ incross progeny heads shown in lateral view, anterior to left. Arrowheads indicate hemorrhage. (H-J) Confocal images of the vasculature (kdrl:EGFP; green) and primitive erythrocytes (gata1:DsRed; magenta) in the same animals as in E-G. Arrowheads indicate hemorrhage. (K,L) Zoomed-in view of central arteries (CtAs; boxed areas in J and K). (M) Quantitation of the percentage of embryos of indicated genotype with hemorrhage by 52 hpf. ${ }^{*} P<0.0003$ compared to WT siblings, by Fisher's exact test with Bonferroni correction. (N-P) Stereoscope transmitted light images of 52 hpf $T g(k d r l: E G F P) ;$ rhoaa ${ }^{\Delta^{3 /+}}$ incross progeny. Arrowhead indicates hemorrhage. (Q-S) Confocal images of the vasculature (kdrl:EGFP; green) of the same animals as in O-Q. Arrows indicate overbranching CtAs. (T,U) Zoomed-in view of CtAs (boxed area in R and S). $(\mathbf{V}, \mathbf{W})$ Quantitation of the percentage of WT sibling incross progeny (left two columns) versus rhoaa $^{\Delta 3 /+}$ incross progeny (right two columns) that either (V) have hemorrhage or lack circulation at $52 \mathrm{hpf}\left({ }^{*} P\right.$ ?? 0.0024 compared to WT incross progeny, by Fisher-Freeman-Halton test), or (W) have reduced sprouting or overbranching of CtAs $\left({ }^{*} P<0.001\right.$, compared to WT incross progeny, by Fisher-Freeman-Halton test). Mean \pm SD is shown for each graph. 


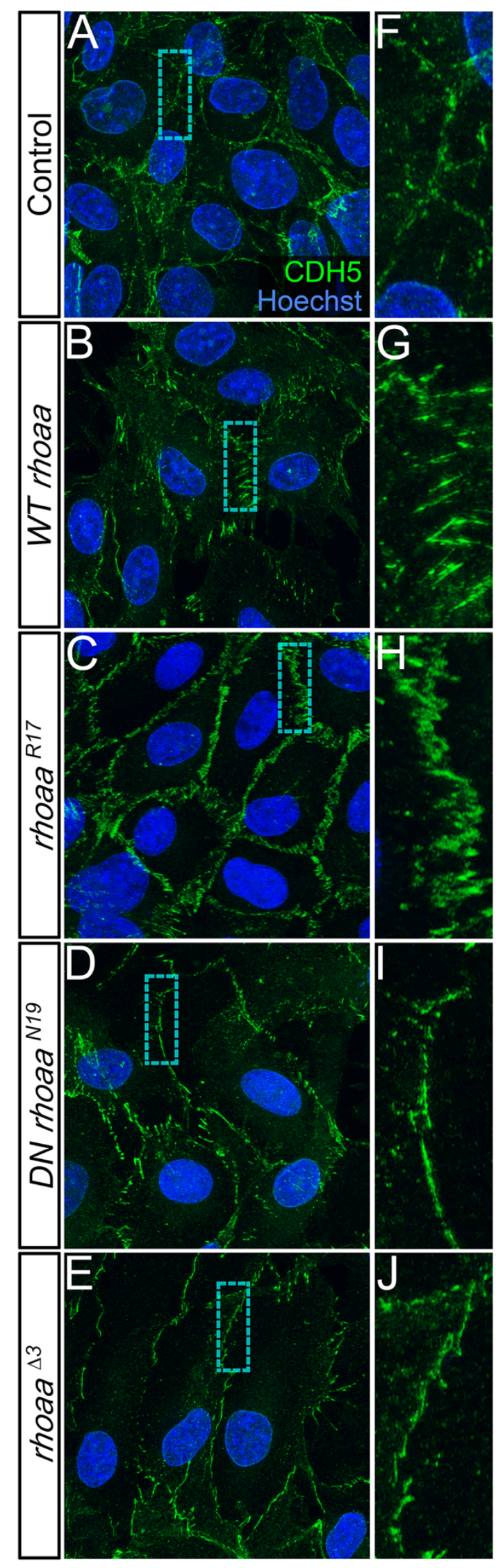

Figure 2. Rhoaa isoforms exhibit distinct activities

in vitro. (A-E) Left: anti-CDH5 immunostaining of adherens junctions (green) in HUVEC stably transfected with wild type (WT) or mutant zebrafish rhoaa isoforms. Nuclei labeled with Hoescht 33342 (blue). (F-J) Zoomed-in view of boxed areas shown in panels on left. 


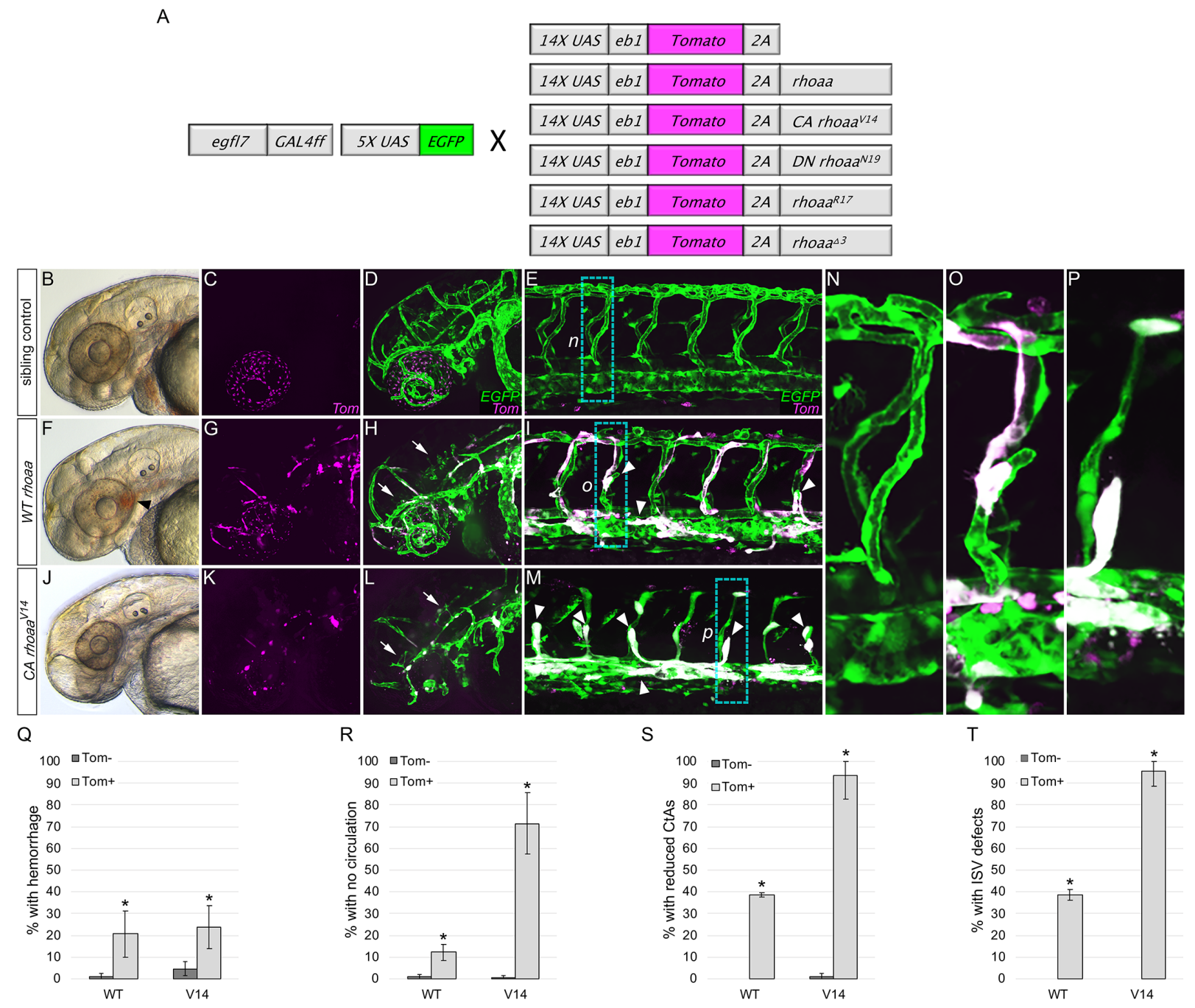

Figure 3. Increased endothelial cell Rhoa activity induces vascular integrity and patterning

defects in vivo. (A) Schematics of transgenics used for conditional wild type or mutant rhoaa expression in zebrafish endothelial cells. $(\mathbf{B}, \mathbf{F}, \mathbf{J})$ Stereoscope transmitted light images of $52 \mathrm{hpf}$ progeny derived from a cross between $T g(e g f l 7: G A L 4 f f), T g(U A S: E G F P)$ and $T g(U A S: T o m a t o-2 A-$ rhoaa) (B,F) or Tg(UAS:Tomato-2A-rhoaav14) (J) fish. Panel B shows a non-Tomato/rhoaa transgene-expressing control sibling of the Tomato/WT rhoaa transgene-expressing animal in panel $\mathrm{F}$. Black arrowhead in $\mathrm{F}$ indicates hemorrhage. (C, D, G, H, K,L) Tomato $(\mathrm{C}, \mathrm{G}, \mathrm{K})$ and Tomato/EGFP $(\mathrm{D}, \mathrm{H}, \mathrm{L})$ confocal images of cranial endothelial cells in the same embryos as in 
panels B,F,J. Arrows indicate reduced CtA sprouting. (E,I,M) Tomato/EGFP confocal images of trunk endothelial cells in 52 hpf progeny derived from a cross between $\operatorname{Tg}($ egfl7:GAL4ff), Tg(UAS:EGFP) and Tg(UAS:Tomato-2A-rhoaa) (E,I) or Tg(UAS:Tomato-2A-rhoaa ${ }^{\text {V14 }) ~(M) ~ f i s h . ~}$ White arrowheads indicate ISV sprouting defects or impaired vessel dilation. (N-P) Magnified Tomato/EGFP confocal images of trunk vasculature in the boxed areas in panels E,I,M. (V-Y) Quantitation of the percentage of 52 hpf Tomato-positive WT Rhoaa-expressing or Rhoaav14_ expressing (light grey columns) animals or their Tomato-negative siblings (dark grey columns) with specified phenotype. Phenotypes shown in the graphs are cranial hemorrhage (Q), lack of circulation (R), reduced cranial CtA sprouting (S), or defective trunk ISV sprouting (T). *P ?< 0.0001 compared to Tomato-negative sibling controls, by Cochran-Mantel-Haenszel test. Mean $\pm S D$ is shown for each graph. 


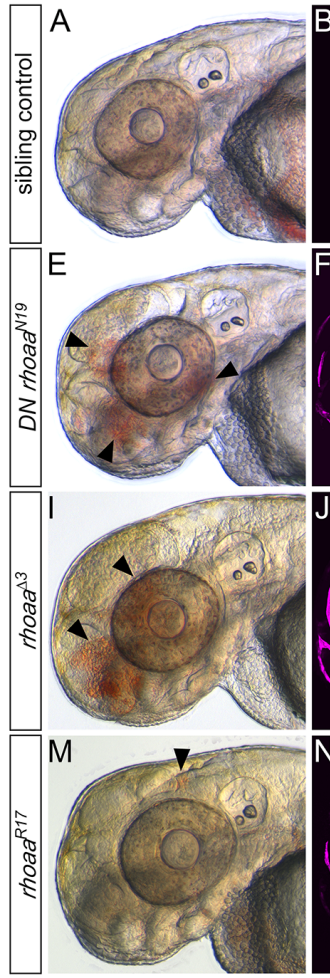

U

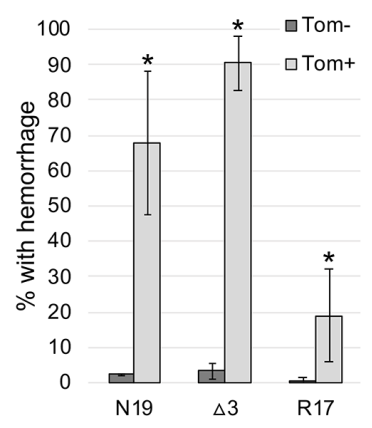

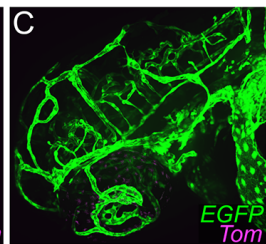
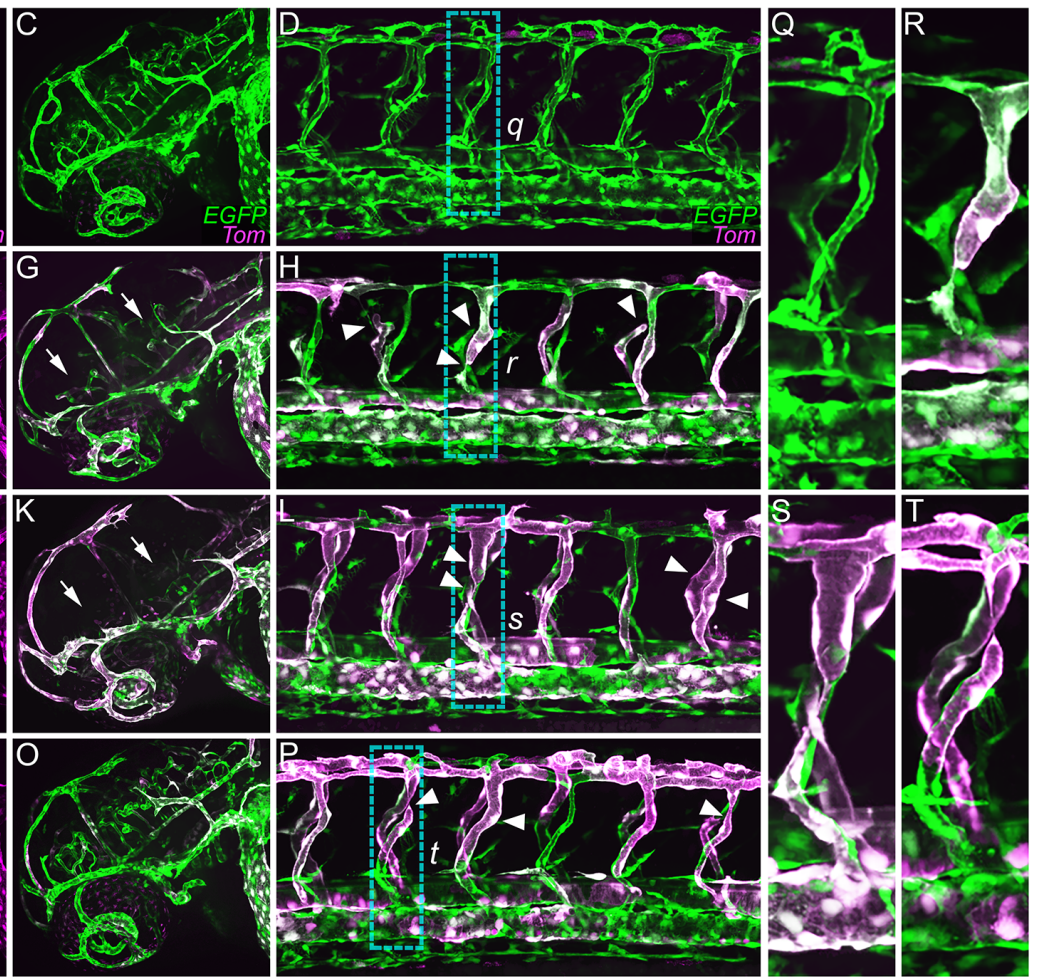

V

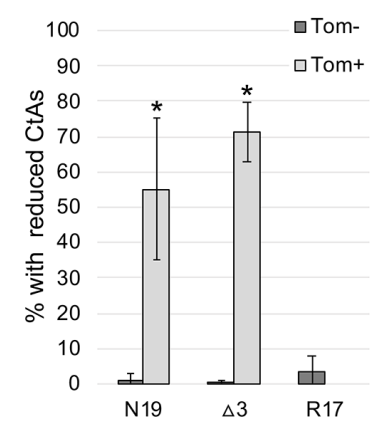

Figure 4. Endothelial cell specific expression of dominant negative rhoaa induces cranial vessel integrity, patterning, and trunk vessel dilation defects in vivo. (A,E,I,M) Stereoscope transmitted light images of 52 hpf progeny derived from a cross between Tg(egfl7:GAL4ff), Tg(UAS:EGFP) and Tg(UAS:Tomato-2A-rhoad $\left.{ }^{\text {N19}) ~(A, E), ~ T g(U A S: T o m a t o-2 A-r h o a a ~}{ }^{3}\right)$ (I), or Tg(UAS:Tomato-2A-rhoaa ${ }^{R 17}$ ) (M) fish. Panel A shows a non-Tomato/rhoaa transgeneexpressing control sibling of the Tomato/rhoaa ${ }^{N 19}$ transgene-expressing embryo in panel $\mathrm{E}$. Black arrowheads in $E$ indicate hemorrhage. (B,C,F,G,J,K,N,O) Tomato $(B, F, J, N)$ and 
Tomato/EGFP $(\mathrm{C}, \mathrm{G}, \mathrm{K}, \mathrm{O})$ confocal images of cranial endothelial cells in the same embryos as in panels $A, E, I, M$. Arrows indicate reduced CtA sprouting. (D,H,L,P) Tomato/EGFP confocal images of trunk endothelial cells in 52 hpf progeny derived from a cross between $T g(e g f / 7: G A L 4 f)$, Tg(UAS:EGFP) and Tg(UAS:Tomato-2A-rhoad $\left.{ }^{N 19}\right)$ (D,H), Tg(UAS:Tomato-2A-rhoaa $\left.{ }^{\Delta^{3}}\right)$ (L), or Tg(UAS:Tomato-2A-rhoaa ${ }^{R 17}$ ) (P) fish. Arrowheads indicate ISV dilation, detachment, or growth defects. (Q,R,S,T) Magnified Tomato/EGFP confocal images of trunk vasculature in the boxed areas in panels $D, H, L, P .(U, V)$ Quantitation of the percentage of Tomato-positive embryos (light grey columns) or their Tomato-negative siblings (dark grey columns) with cranial hemorrhage (U) or reduced cranial CtA sprouting (V) at $52 \mathrm{hpf.}{ }^{*} P ?<0.0001$ compared to Tomato-negative sibling controls, by Cochran-Mantel-Haenszel test. Mean \pm SD is shown for each graph. 

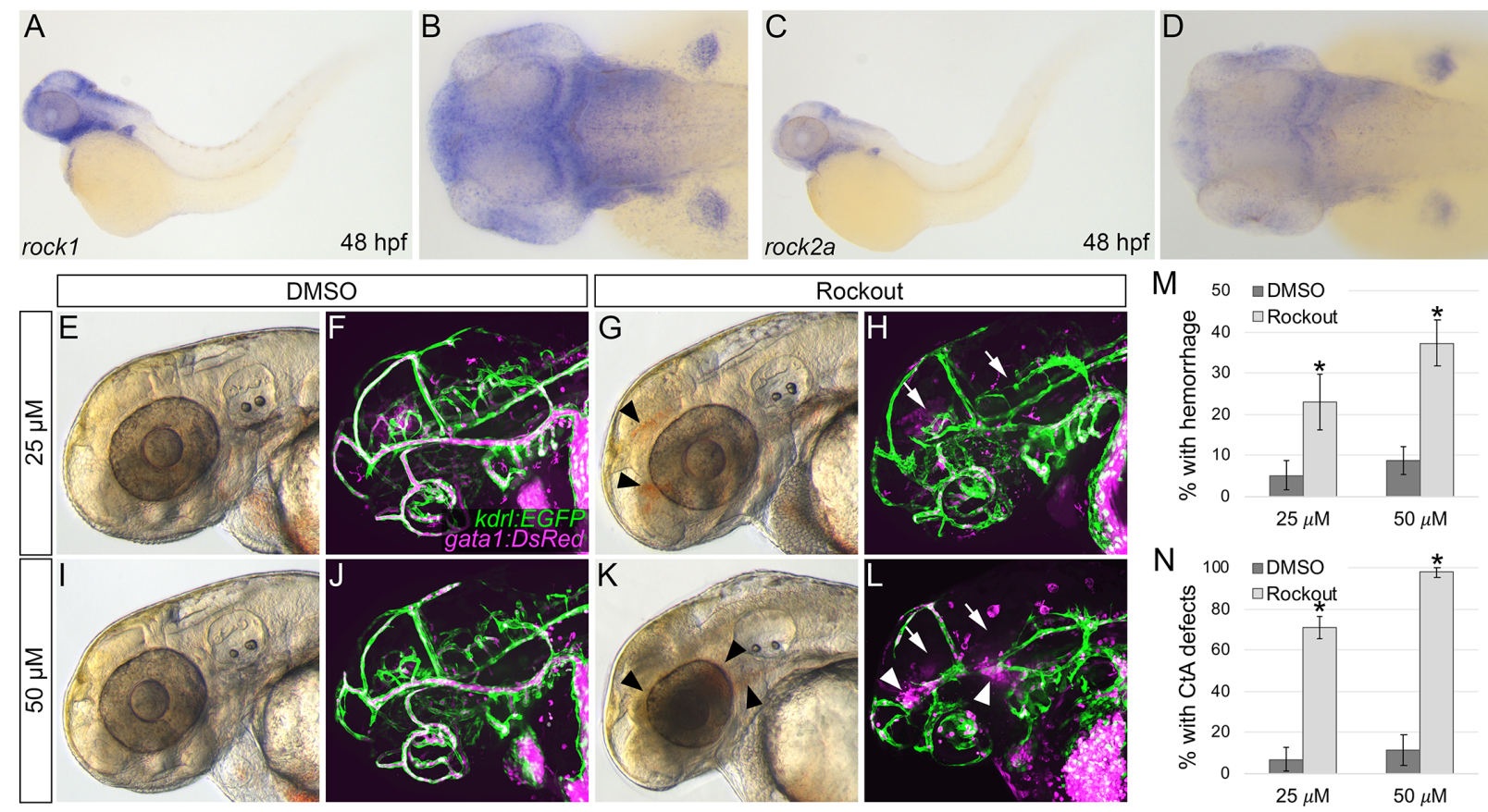

Figure 5. Pharmacological inhibition of Rhoa effectors Rock1/2 produces cranial hemorrhage and cranial angiogenesis defects. (A-D) Whole-mount in situ hybridization analyses of $48 \mathrm{hpf}$ wild type zebrafish embryos probed for rock1 $(A, B)$ or rock2a $(C, D)$ gene expression. $(A, C)$ Lateral views, anterior to left. $(B, D)$ Higher magnification dorsal views of the heads shown in panels A,C. $(\mathbf{E}, \mathbf{G}, \mathbf{I}, \mathbf{K})$ Stereoscope transmitted light images of DMSO-treated control sibling $(E, I)$ or $25 \mu \mathrm{M}(\mathrm{G})$ or $50 \mu \mathrm{M}(\mathrm{K})$ Rock1/2 inhibitor (Rockout)-treated $50 \mathrm{hpf} \mathrm{Tg}(\mathrm{kdrl}: E G F P)$, Tg(gata1a:DsRed) embryos. Arrowheads indicate hemorrhage. (F,H,J,L) Confocal images of cranial vessels (EGFP-positive, green) and blood cells (DsRed-positive, magenta) in the same embryos shown in panels $E, G, I, K$. Arrows indicate CtA defects, arrowheads indicate hemorrhage. (M,N) Quantitation of the percentage of $52 \mathrm{hpf}$ Rockout-treated (light grey columns) or DMSO-treated (dark grey columns) sibling embryos with cranial hemorrhage (M) or cranial CtA defects (N). ${ }^{*} P<0.0001$ compared to DMSO-treated controls, by Cochran-MantelHaenszel test. Mean \pm SD is shown for each graph. 


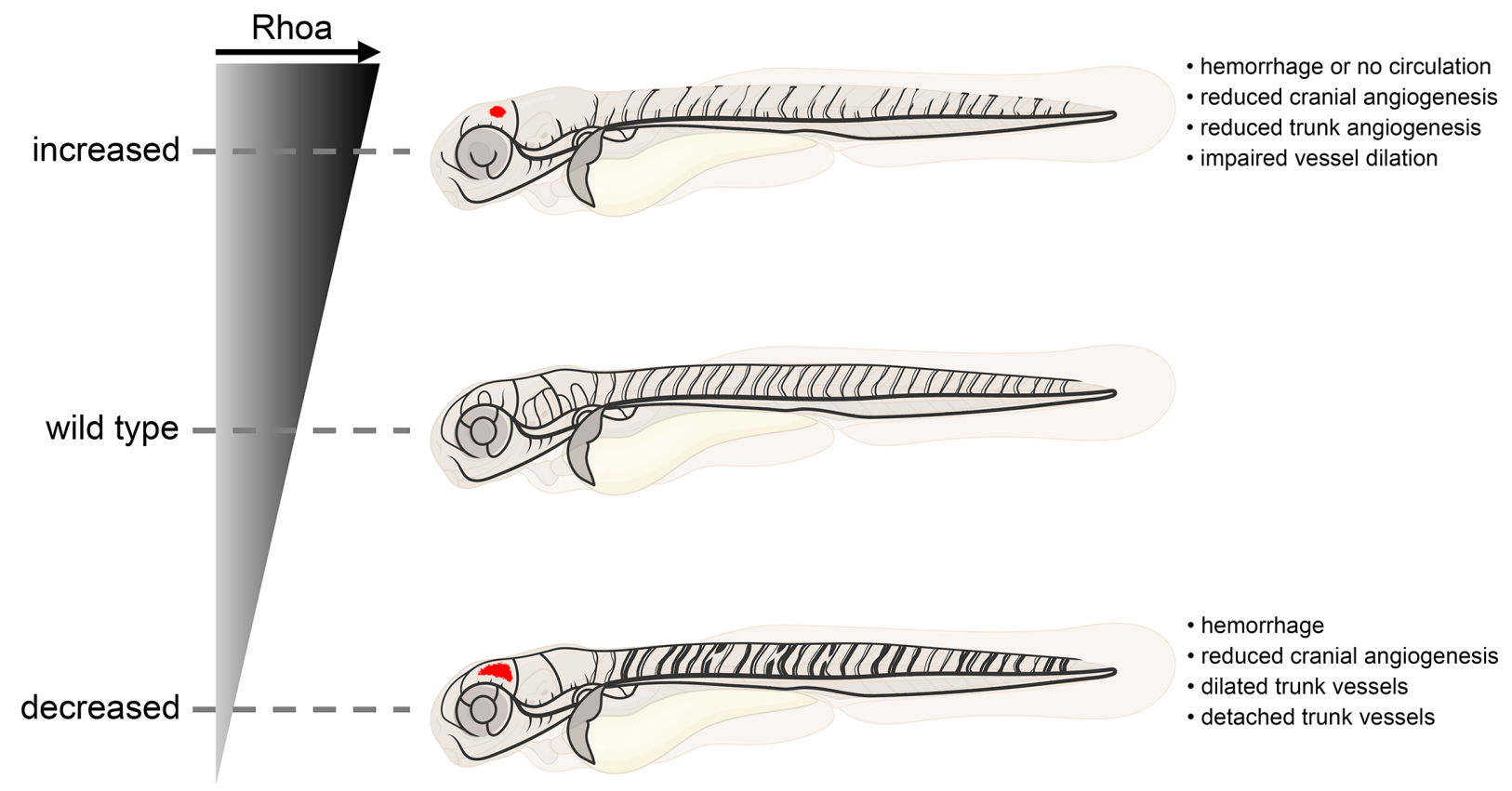

Figure 6. Summary of embryonic vascular defects generated by increased or decreased Rhoa

signaling. Schematic diagrams illustrating the range of different vascular defects found in 52 hpf zebrafish embryos with either increased, wild type, or decreased endothelial cell levels of Rhoa activity. Embryos drawn in lateral view, anterior to the left, with blood vessels in black and cranial hemorrhage in red. 